\title{
Unaliasing by Fourier-Encoding the Overlaps Using the Temporal Dimension (UNFOLD), Applied to Cardiac Imaging and fMRI
}

\author{
Bruno Madore, ${ }^{*}$ Gary H. Glover, and Norbert J. Pelc
}

\begin{abstract}
In several applications, MRI is used to monitor the time behavior of the signal in an organ of interest; e.g., signal evolution because of physiological motion, activation, or contrast-agent accumulation. Dynamic applications involve acquiring data in a $k-t$ space, which contains both temporal and spatial information. It is shown here that in some dynamic applications, the $t$ axis of $k-t$ space is not densely filled with information. A method is introduced that can transfer information from the $k$ axes to the $t$ axis, allowing a denser, smaller $k-t$ space to be acquired, and leading to significant reductions in the acquisition time of the temporal frames.

Results are presented for cardiac-triggered imaging and functional MRI (fMRI), and are compared with data obtained in a conventional way. The temporal resolution was increased by nearly a factor of two in the cardiac-triggered study, and by as much as a factor of eight in the fMRI study. This increase allowed the acquisition of fMRI activation maps, even when the acquisition time for a single full time frame was actually longer than the paradigm cycle period itself.

The new method can be used to significantly reduce the acquisition time of the individual temporal frames in certain dynamic studies. This can be used, for example, to increase the temporal or spatial resolution, increase the spatial coverage, decrease the total imaging time, or alter sequence parameters e.g., repetition time (TR) and echo time (TE) and thereby alter contrast. Magn Reson Med 42:813-828, 1999. (c) 1999 WileyLiss, Inc.
\end{abstract}

Key words: cardiac imaging; fMRI; reduced FOV; rapid imaging; dynamic imaging

Several applications of MRI involve acquiring a time series of images in order to resolve the variations experienced by the imaged object. These dynamic studies include cardiac imaging (1-6), functional MRI (fMRI) (7-9), time-resolved angiography (10), and contrast agent uptake studies $(6,11-$ 15). When dynamic objects are imaged, an ideal approach would completely fill $k-t$ space (16), thereby providing all the desired spatial information at any moment in time. Because the imaging process is often too slow to acquire all this information, methods have been developed that acquire only a part of the desired $k-t$ space, the rest being obtained through some model or approximation. Such

Lucas MRS Center, Department of Radiology, Stanford University School of Medicine, Stanford, California.

*Correspondence to: Bruno Madore, Stanford University School of Medicine, Lucas MRS Center, Department of Radiology, 1201 Welch Rd, Stanford, CA 94305-5488. E-mail: bruno@s-word.stanford.edu

Grant sponsor: National Institutes of Health; Grant number: P41 RR09784; Grant sponsors: NSERC; GE Medical Systems; the Lucas Foundation.

Received 28 December 1998; revised 13 April 1999; accepted 18 May 1999. 1999 ISMRM Young Investigators' W.S. Moore Award Winner.

(C) 1999 Wiley-Liss, Inc. methods include keyhole imaging (17-20), reducedencoding MR imaging with generalized-series reconstruction (RIGR) (21-23), data sharing (24-28), zero filling (29), reduced field-of-view (FOV) methods, which assume that much of the imaged object (with the exception of a dynamic region) is static (30-33) or undergoing a cyclic motion (33), and non-Fourier approaches such as singular value decomposition (SVD) and wavelet methods (34-37). With non-Fourier approaches, the acquired data does not belong to the $k-t$ space but is used in the calculation of a full $k-t$ space.

These earlier methods and the work presented here share the same goal: to reduce the amount of data needed for a given spatial and temporal resolution. UNaliasing by Fourier-encoding the Overlaps using the temporaL Dimension (UNFOLD) is a new, flexible way of encoding spatiotemporal information with MRI. Inefficiencies in some dynamic applications are identified, and the extra flexibility introduced here makes it possible to partially avoid such inefficiencies. The reductions in data requirements (up to nearly a factor two with cardiac imaging, and as much as a factor eight with fMRI) comes from a more efficient encoding of the desired information.

Like other methods (30), one of the first steps in UNFOLD involves a reduction of the dynamic FOV. This FOV reduction diminishes the amount of spatial information acquired along the $k$ axes of $k-t$ space. Because of aliasing, spatially distinct points within the object are overlapped at a same spatial position in the images. UNFOLD then uses time to label the overlapped components, such that a Fourier transform through time can resolve them. This use of the time axis to encode some spatial information introduces a certain freedom in deciding "what should be encoded where" in $k-t$ space. In some applications, $k-t$ space can be thought of as a partially filled box, having free spaces in hard-to-reach areas. For example, in cardiac imaging the most dynamic changes might be confined to only a part of the FOV, while in fMRI signal variations are expected only at temporal frequencies dictated by the paradigm. In such cases, the ability to transfer some information from a $k$ axis to the $t$ axis may be sufficient to build a tighter, denser, and smaller $k-t$ space allowing the acquisition of fewer data points. As will be shown, such reformatting of $k-t$ space may lead to significant reductions in data requirements.

The theory behind UNFOLD is presented as follows. First, it is shown that time can be used to label aliased spatial components. Different imaging methods give rise to 
different types of aliasing. For this reason, the theory is first presented in the context of Cartesian FT imaging, where aliasing consists of a simple overlap of what should be distinct spatial points. Then, a more general approach is presented where the spatial aliasing may be more complicated. Specific applications, such as cardiac imaging and fMRI, are then considered.

\section{THEORY}

UNFOLD provides a way of discriminating between spatially aliased and nonaliased components in images. Accordingly, two or more object points can be deliberately overlapped through aliasing, and separated afterward using the method described herein. In some circumstances, described in detail later in this report, such a scheme leads to efficient ways of encoding dynamic information, diminishing significantly the amount of data that is required to achieve a given spatial and temporal resolution.

\section{How to Differentiate Aliased from Non-Aliased Material by Using Time Modulations}

\section{Data Sampled on a Cartesian Grid (Fourier Transform Imaging Methods)}

When an object is imaged, only certain points in $k$-space have their value actually measured. The "sampling function" $(S(\vec{k}))$ represents how $k$-space is sampled by a given imaging method: it is a delta function at the $\vec{k}$ locations that are sampled, and zero elsewhere. The relationship between the object $O(\vec{r})$, its image $I(\vec{r})$, and the sampling function is given by:

$$
I(\vec{r})=\mathscr{F}(S(\vec{k})) * O(\vec{r})=P S F(\vec{r}) * O(\vec{r})
$$

where $\vec{r}$ and $\vec{k}$ are position-vectors in, respectively, the object domain and $k$-space, ' $\mathscr{F}$ ' represents a Fourier transform $(\mathrm{FT})$, * represents a convolution, and $\operatorname{PSF}(\vec{r})=$ $\mathscr{F}(S(\vec{k}))$ is the point-spread function (for simplicity, other contributions to the PSF, like $\mathrm{T}_{2}$ and $\mathrm{T}_{2}^{*}$, are left out). Figure 1a depicts a sampling function $S\left(k_{x}, k_{y}\right)$ as used by 2D DFT imaging methods. With DFT methods, $k$-space data is sampled directly on a Cartesian grid. (The $k_{x}$ lines in Fig. 1a are depicted as continuous lines rather than as a series of points for simplicity and because the sampling along $k_{X}$ is assumed sufficient to avoid aliasing in the $x$ direction.) The PSF associated with Cartesian sampling, the FT of Fig. 1a, is shown in Fig. 1b (its peaks are numbered for future reference). Through the convolution in Eq. [1] the multiple peaks of the PSF replicate the object $O(x, y)$ in the $y$ direction. The result is shown in Fig. 1c. The two dashed lines in Fig. 1c depict $\mathrm{FOV}_{y}$, the FOV in the $y$ direction. These lines are located half way between the 0th and the \pm 1 st peak of the PSF shown in Fig. 1b. If the imaged object is contained within these two lines, then no aliasing occurs.

Figure 2 shows the result when the number of lines in the sampling function is reduced by a factor two, given the same object and spatial bandwidth as in Fig. 1. This change in the sampling function reduces $\mathrm{FOV}_{y}$ by a factor two, resulting in the well-known problem of aliasing $(38,39)$.

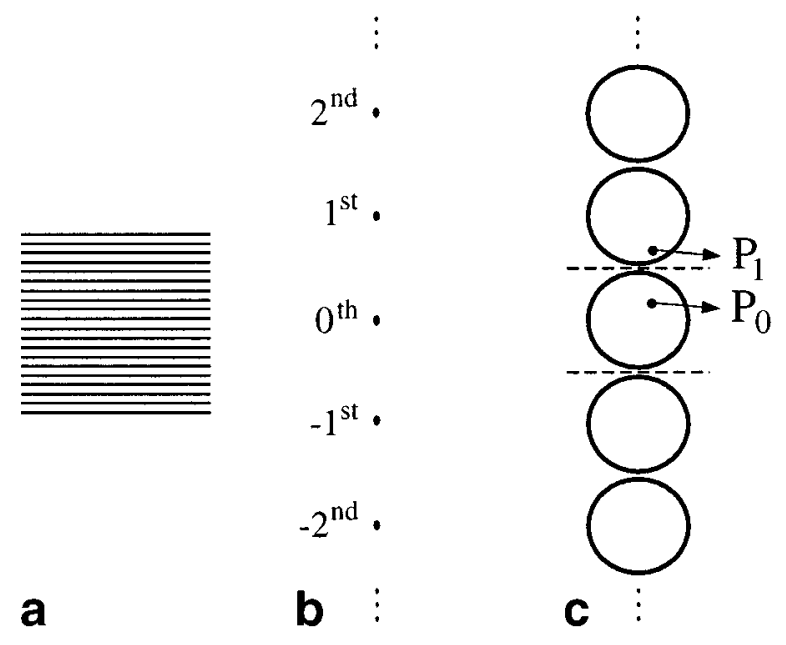

FIG. 1. Graphical representation of Eq. [1]. The Cartesian sampling function $S\left(k_{x}, k_{y}\right)$ shown in a gives rise, through an $\mathrm{FT}$, to the PSF shown in $\mathbf{b}$. The multiple peaks of the PSF replicate an object (here a circle) through the convolution in Eq. [1], as shown in c. Two points are shown in c: $P_{0}$ lies in the replica created by the 0th peak of the PSF, whereas $P_{1}$ lies in the replica coming from the 1st peak. $P_{0}$ and $P_{1}$ are separated by $F O V_{y} / 2$.

Object points overlap, as shown in Fig. 2c: the points $P_{0}$ and $P_{1}$, which were separated in Fig. 1c, are now overlapped into a single image point in Fig. 2c.

The idea behind UNFOLD is introduced in Fig. 3. UNFOLD involves shifting the sampling function in the phase-encoding direction. From the shift theorem (40), a shift of $S(\vec{k})$ by a fraction $f$ of a line (as depicted in Fig. 3a) results in a linear phase shift being applied to $P S F(\vec{r})$, (as depicted in Fig. 3b), altering the phase of all but the central peak. Through the convolution in Eq. [1], the phase of each PSF peak is passed to the corresponding replica of the object as shown in Fig. 3c. For example, the point $P_{0}$ remains unchanged by the shift (because it originates from the central peak of the PSF), but the point $P_{1}$ is phase shifted by an angle $2 \pi f$ (because it originates from the first peak of the PSF). In a dynamic study, where several images of an object are acquired, the shift in the sampling function can be varied from image to image. This time-varying shift

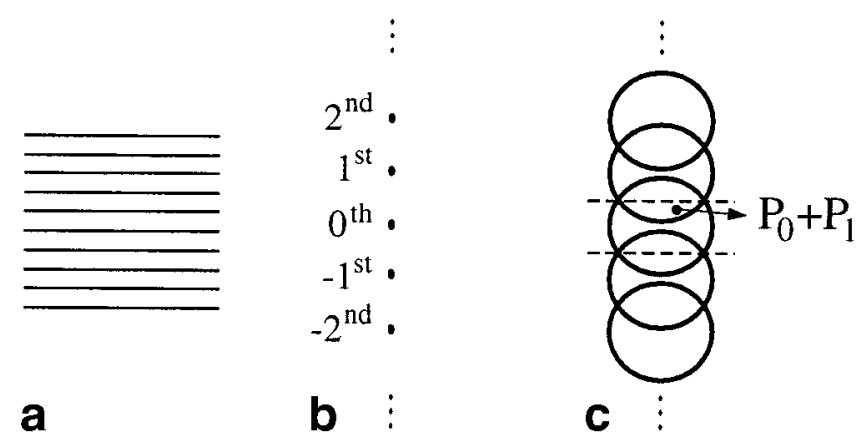

FIG. 2. Distance between the lines of the sampling function in a is increased by a factor of two compared with Fig. 1. As a result, the distance between adjacent peaks of the PSF is reduced by a factor of two in $\mathbf{b}$, and the replicas of the object overlap in $\mathbf{c}$. Because of aliasing, the points $P_{0}$ and $P_{1}$ that were distinct in Fig. 1c are overlapped in c. 
FIG. 3. a: The sampling function shown in Fig. 2 is shifted along the phase-encoding direction. Dashed and full lines represent, respectively, the old and new location of the sampling function. Through the shift theorem, this shift generates a phase ramp in the PSF shown in $\mathbf{b}$. Phase shifts at the PSF peaks are passed to the replicas in c through the convolution in Eq. [1].

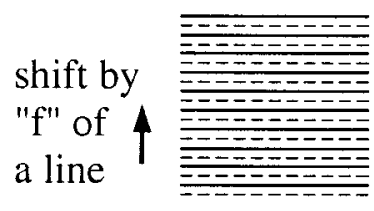

a

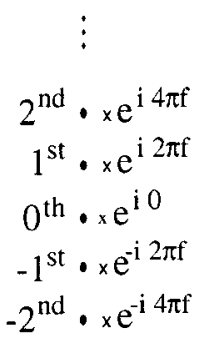

b :

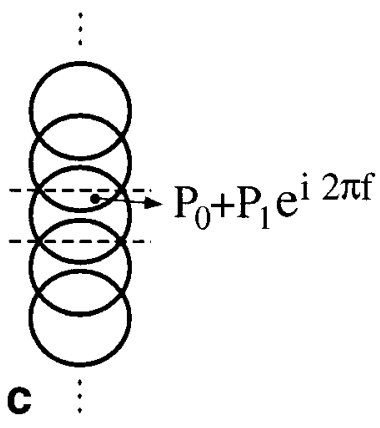

can be used to "label," and then resolve, the various components that are overlapped, by modulating their phase as a function of time in a controlled way.

Consider the following example. Assume that both $P_{0}$ and $P_{1}$ are constant in time. A time series of images is acquired, where every odd image in the series is obtained using a sampling function shifted by half a line $(f=0.5)$ compared with that used for the even images. As a result, the aliased components in the odd images are multiplied by $e^{i 2 \pi f}=-1$ (Fig. 4a). More specifically, the value of the image point where $P_{0}$ and $P_{1}$ overlap oscillates between
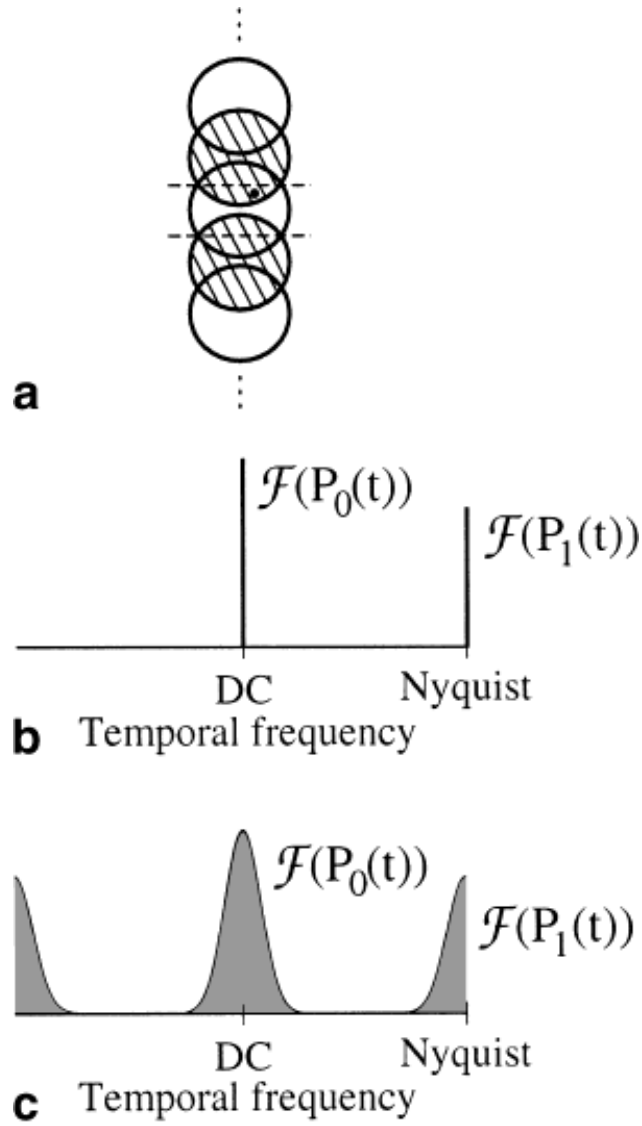

FIG. 4. a: A shift of half a $k_{y}$ line changes the sign of every odd-ordered replica (hatched circles). b: In a time series where $f$ (shown in Fig. 3) oscillates between 0 and 0.5 every second frame, the point $P_{0}$ (shown in Figs. 1c and 3c) remains constant through time while the point $P_{1}$ changes sign at the Nyquist frequency. c: If $P_{0}$ and $P_{1}$ are time-dependent, their temporal frequency spectrum becomes a distribution of frequencies instead of a delta function.
$P_{0}+P_{1}$ (even images) and $P_{0}-P_{1}$ (odd images). This is reminiscent of some ghost-removing methods developed for EPI $(41,42)$, where certain problems at the acquisition stage can generate inconsistencies between even and odd lines. Reconstructing these separately may help in finding parameters needed for accurate reconstruction. Here, the focus of interest is the dynamic nature of the imaged object, rather than the effect of hardware imperfections.

Figure $4 \mathrm{~b}$ shows the temporal frequency spectrum (the FT in the time direction) of the signal at the image point where $P_{0}$ and $P_{1}$ overlap. The spectrum contains a component at the Nyquist frequency (the point $P_{1}$ ) and a DC component (the point $P_{0}$ ). Notice that $P_{0}$ and $P_{1}$ in Fig. $4 \mathrm{~b}$ are no longer overlapped; they have been Fourier encoded to different locations in the temporal frequency domain, and they can be separated in Fig. 4b as surely as in Fig. 1c (where $P_{0}$ and $P_{1}$ are Fourier encoded to different locations in the spatial domain).

In Fig. 4c, the assumption that $P_{0}$ and $P_{1}$ are constant in time is removed. The spectrum associated with the point $P_{0}(t), \mathscr{F}\left(P_{0}(t)\right)$, now contains a range of frequencies instead of a single (DC) component. The more "dynamic" a point is, the wider is the frequency range required to describe its time variations. The same can be said of $P_{1}(t)$ and its spectrum $\mathscr{F}\left(P_{1}(t)\right)$. As in Fig. $4 \mathrm{~b}$, the spectrum associated with $P_{1}$ is shifted by half the temporal bandwidth and centered at the Nyquist frequency. This is because of the shifts applied to the sampling function, which change the sign of $P_{1}$ every second frame. Since the spectra from $P_{0}$ and $P_{1}$ are separated in Fig. 4c, the time dependence of one point can be obtained by filtering out (i.e., removing) the spectrum associated with the other point and applying a Fourier transform to the result.

The example presented above can be further generalized. Imagine $n$ points overlapped into a single voxel through spatial aliasing. These $n$ points are referred to as $P_{j}$, where $j$ is the order of the PSF peak from which a point originates. The value of the resulting composite point is given by

$$
P(t)=\sum_{j=-f l o o r((n-1 / 2))}^{\text {floor }(n / 2)} P_{j}(t) \cdot e^{i 2 \pi f(t) j}
$$

where floor( ) rounds a real number to the nearest lower integer and $f(t)$ is the $k$-space shift of the sampling function $S(\vec{k})$ employed at time $t$. A useful special case to consider 
for $f(t)$ is a linear function

$$
f(t)=\frac{t}{n}
$$

where the time variable $t$ is actually the image number in a time series. Substituting Eq. [3] into Eq. [2] yields

$$
P(t)=\sum_{j=- \text { floor }((n-1 / 2))}^{\text {floor }(n / 2)} P_{j}(t) \cdot e^{i 2 \pi t j / n}
$$

The complex exponential in Eq. [4] is in fact a Fourier basis function. The temporal frequency spectrum $\mathscr{F}(P(t))$ contains $n$ peaks, uniformly spaced across the bandwidth. Each peak is associated with one of the $n$ spatially overlapped points. The example in Fig. 4c represents the case $n=2$ ( $n=2$ in Eq. [3] leads to $f$ being successively $0,1 / 2,1$, $3 / 2,2, \ldots$ while in Fig. 4, the pattern was $0,1 / 2,0,1 / 2$, $0, \ldots$; the two patterns are equivalent, because of the cyclic nature of angles and of the complex exponential.).

A larger measured temporal bandwidth may be required if, instead of one spectrum, $n$ spectra were to be placed side by side in the bandwidth with a minimum of overlap. Clearly, UNFOLD becomes useless if the only way to fit $n$ spectra without overlap involves increasing the bandwidth by a factor $n$. The idea behind this report is that in certain applications, forcing two or more points to share the same temporal bandwidth leads to more efficient ways of encoding information. Two such cases, (cardiac imaging and fMRI), are presented later.

We have shown that spatial points that are overlapped because of spatial aliasing can be resolved by applying shifts to a Cartesian sampling function. As described next, this method can also be used with non-Cartesian sampling trajectories.

\section{Data Sampled by Using an Arbitrary Trajectory}

Consider a time series of images acquired using an arbitrary sampling trajectory in $k$-space. The chosen trajectory is assumed to provide an adequate sampling for the desired spatial resolution and FOV. Instead of acquiring all the $k$-space samples for every time frame, only a fraction of the samples is collected (e.g., a fraction of all the $k_{y}$ lines in a Cartesian FT scan, or a fraction of the interleaves in a spiral (43) scan). This partial coverage of $k$-space gives rise to individual time frames that may be corrupted by spatial aliasing. From one time frame to the next, a different set of $k$-space points is acquired, such that a full $k$-space matrix is collected in $n$ time frames. The sampling functions used for the $n$ frames are then used again, in a periodic way, to acquire the subsequent images in the time series.

Suppose that an object, assumed for now to be static in time, is imaged using the scheme described above. A time series of seemingly corrupted images is generated, where the intensity at every image point is a periodic function of time with a period of $n$ time frames. As stated previously, the sum of the sampling functions used for $n$ consecutive time frames is adequate for the desired spatial resolution and FOV. Using the linearity of the Fourier transform, the sum of $n$ consecutive time frames is a noncorrupted image. In other words, the signal in every voxel in every time
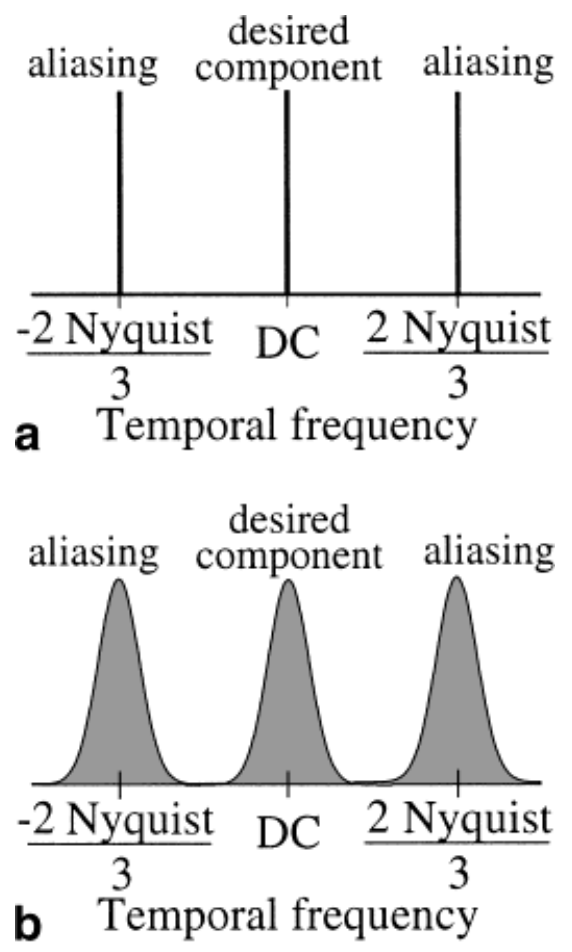

FIG. 5. a: If the imaged object is static in time, the temporal modulation generated at a given voxel by the use of three different sampling functions gives rise to three delta functions in the temporal frequency domain: one at DC, one at $2 / 3$ Nyquist, and one at $-2 / 3$ Nyquist. Component at DC contains the information about the non-aliased object while the two others contain the signal that is spatially aliased. b: If the imaged object is dynamic, a range of temporal frequencies is required to describe the aliased and nonaliased components.

frame consists of the correct value, plus an error component coming from the insufficient sampling of $k$-space. However, the temporal DC component of this error term is zero. Indeed, the temporal average of the time frames is a nonaliased image.

The Fourier transform of the time-varying intensity at a given voxel has no more than $n$ nonzero frequency components, including a DC component. This is derived from the fact that the voxel intensity varies with a period of $n$ time frames. An example is given in Fig. 5a for $n=3$, where three nonzero frequency components can be found uniformly spaced in the temporal frequency domain. The UNFOLD approach is based on the fact that the desired and aliased components are encoded at different locations in the temporal frequency domain, so the desired component can be recovered. As shown in Fig. 5b, if the imaged object is dynamic, a range of temporal frequencies is required to describe the aliased and the non-aliased components.

The situation depicted in Fig. 4 is a special case of the method described here. The $k$-space sampling function is Cartesian, the full sampling function is divided into $n$ parts by selecting a $k_{y}$ line very $n$ lines, and the partial sampling functions are simple translations of one another. This simplicity gives rise to especially convenient behavior in the spatial domain, as the aliasing simply consists of an overlap of $n$ object points at every image point. In more general cases, the aliased components contained in the non-DC peaks may come from a large number of object 
points. As will be shown in the following sections, the cardiac application may require UNFOLD to have a simple spatial behavior, but the fMRI application does not have such a requirement. Accordingly, a Cartesian imaging sequence was used to acquire the cardiac results, while a non-Cartesian one (spiral imaging) was used to acquire the fMRI results.

\section{Applications for the UNFOLD Method}

UNFOLD allows the time axis to be used to encode some spatial information. In a case where every spatial point in the FOV needs the full temporal bandwidth to be adequately described, UNFOLD would provide no advantage. However, if some spatial points do not require the full bandwidth, then it may be possible to efficiently "stack" or interleave more than one spatial point in what would otherwise be the full bandwidth of a single image point. The following paragraphs show how such an encoding scheme can significantly reduce the data requirements in cardiac-triggered imaging and fMRI.

Objects With Highly Dynamic and Less Dynamic Regions (Example: Cardiac-Triggered Imaging)

In general, the signal at every point in the FOV could vary as a function of time, because of cardiac or respiratory motions, or the transit of a contrast agent. However, the signal in some regions could be much more variable than that in others. For example, in cardiac-gated imaging, proper characterization of the signal in the heart could require a much higher temporal bandwidth than is needed for the chest wall. Some methods presented in the past are successful when the imaged object is composed of a portion that is static (or moving in a predictable way (33)), and another portion which is dynamic (30-33). However, these methods are not appropriate if the anatomy outside the dynamic region of interest (ROI) is almost static, but not static. UNFOLD has the ability to handle a situation such as imaging of a dynamic heart surrounded by less dynamic (but not static) material.

Because its complex intensity may vary rapidly in time, the temporal frequency spectrum of a pixel in the highly dynamic portion can be relatively wide, as depicted in Fig. 6a. On the other hand, pixels outside the heart might be expected to have a narrower spectrum, as depicted in Fig. $6 \mathrm{~b}$. Notice that a large portion of the bandwidth $B W_{1}$ in Fig. $6 \mathrm{~b}$ is left unused. Fig. 6c depicts the solution offered by the present method to encode information more efficiently. Each wide (highly dynamic) spectrum, as shown in Fig. 6a, is coupled with a narrower (less dynamic) spectrum, as shown in Fig. 6b. By using the method described previously, they are forced to share the same bandwidth (i.e., the same image voxel). A bandwidth $B W_{2}$, possibly larger than $B W_{1}$, is used to accommodate both spectra. These bandwidths $B W_{1}$ and $B W_{2}$ refer to the variation in the signal at a single image point and should not be confused with the data acquisition bandwidth used during the measurement of a single free induction decay (FID) or echo.

The following two examples give a more practical view of how the present method could be used. Consider a given application generating a time frame every $T$ sec leading to a temporal bandwidth $B W_{1}=1 / T \mathrm{~Hz}$. Because UNFOLD
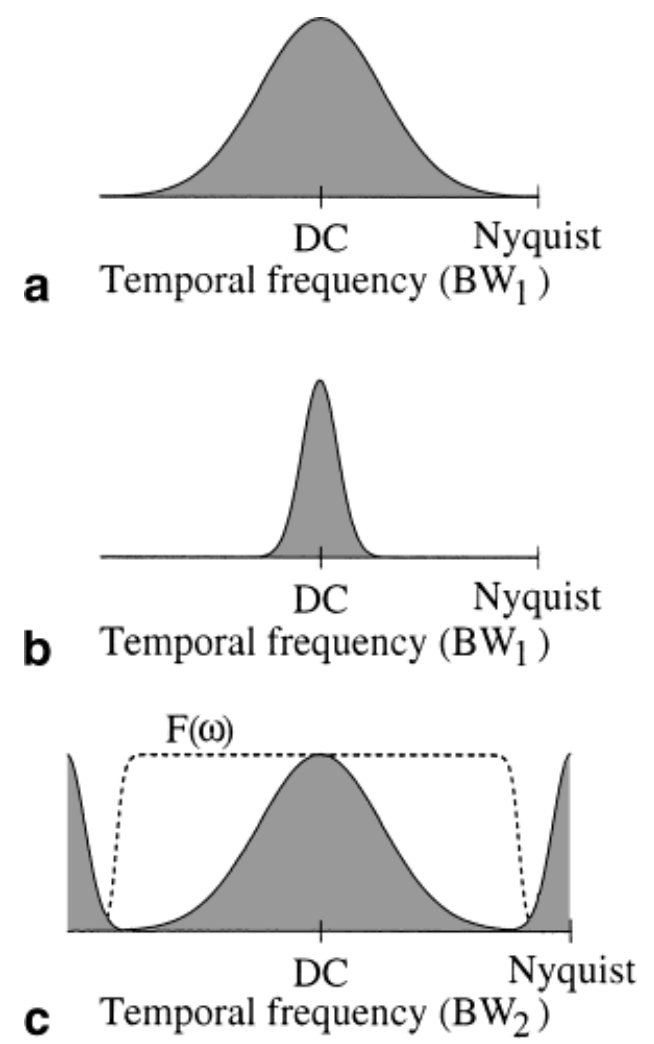

FIG. 6. a: Temporal frequency spectrum of a voxel containing (dynamic) heart material. b: Spectrum of a less dynamic voxel. UNFOLD achieves reductions in data requirements by avoiding the acquisition of nearly empty spectra like the one in b. c: Approach proposed here to encode data more efficiently. The two spectra combined in $\mathbf{c}$ can be separated at the postprocessing stage using a filter such as $F(\omega)$, shown with a dashed line in $\mathbf{c}$.

halves the amount of data (e.g., number of $k_{y}$ lines) required to generate a time frame, one can double the number of time frames per unit time (the bandwidth becomes $B W_{2}=2 B W_{1}$ ) without changing the imaging parameters (e.g., same TR). Because most of the bandwidth $B W_{2}$ can be devoted to the wider spectrum, this approach nearly doubles the temporal resolution for the dynamic signal. Another way of using UNFOLD would consist in keeping the number of frames per unit time constant $\left(B W_{2}=B W_{1}\right.$; the temporal resolution in the dynamic region remains nearly the same), while acquiring more elaborate frames (e.g., double the spatial resolution, or double TR). Any advantage offered by UNFOLD (an increase in temporal resolution $\left(B W_{2}=2 B W_{1}\right)$, more elaborate time frames $\left(B W_{2}=B W_{1}\right)$ or a combination of the two $\left(B W_{1} \leq B W_{2} \leq\right.$ $\left.2 B W_{1}\right)$ ) is obtained by encoding useful information into areas that would otherwise be left empty, such as most of the bandwidth in Fig. $6 \mathrm{~b}$.

Two wide temporal frequency spectra (like the one in Fig. 6a) should not be coupled in the same bandwith. If all of the most dynamic points are localized within a certain area of the object (like the region of the heart), an acquisition FOV is selected that is at least as large as the dynamic region. Although the object itself may be larger than the acquisition FOV, having the dynamic region no bigger than the acquisition FOV guarantees that no aliasing from a dynamic point can overlap onto another dynamic point. 
The two spectra sharing the same bandwidth in Fig. 6c can be separated from each other using a filter $F(\omega)$, as represented by a dashed line in Fig. 6c. This filter increases the $S N R$, by a factor $f_{S N R}$ which depends on the filter, since it removes the noise contained in part of the $B W_{2}$ bandwidth

$$
f_{S N R}=\sqrt{\frac{B W_{2}}{\int_{B W_{2}} F^{2}(\omega) \mathrm{d} \omega}}\left(\frac{V_{2} \sqrt{\tau_{2}}}{V_{1} \sqrt{\tau_{1}}}\right)
$$

The index 1 refers to a conventional acquisition in which the whole (unaliased) FOV is imaged with a same temporal resolution, whereas the index 2 refers to an UNFOLD application. $V$ and $\tau$ represent, respectively, the volume of a voxel and the time spent acquiring data for a time frame. The term on the right hand side involving $V$ s and $\tau$ s comes from well-known relationships for the signal-to-noise ratio (SNR) in MRI (44). The first term on the right hand side accounts for the effect of the filter. Eq. [5] is not valid if UNFOLD is used to change the imaging parameters (e.g., TR), because the effect on SNR would then be a function of pulse sequence timing as well as relaxation times. To preserve the peak at the Nyquist frequency and suppress the one at DC (instead of the inverse), a filter $[1-F(\omega)]$ is used instead of $F(\omega)$. Because [1 $-F(\omega)]$ is narrower than $F(\omega)$, it removes more noise and as a result, in any single image, the SNR will be higher in the less dynamic region than in the more dynamic one. This is exactly as was observed and explained elsewhere for the case in which the less dynamic region is fully static (30). In the case in which UNFOLD would be used to increase the temporal resolution in the dynamic part, we have $B W_{2}=2 B W_{1}, V_{2}=$ $V_{1}$, and $\tau_{2}=\tau_{1} / 2$ (only half the $k_{y}$ lines are acquired for any given frame). If one of the two peaks is narrow, then the filter used is equal to 1 over nearly the whole bandwidth $B W_{2}$, and Eq. [5] gives $f_{S N R} \simeq 1 / \sqrt{2}$. In such a case, UNFOLD doubles the number of time frames while reducing by about $\sqrt{2}$ the SNR in the dynamic portion of any individual frame. If one of the peaks is so narrow that it can be considered a delta function, the present method becomes completely equivalent to the method described by Frederickson and Pelc (30), with the same time savings and SNR properties. Although the example used here is cardiac imaging, the present method could be applied to other time series of images where highly dynamic changes are localized within only part of the imaged object.

\section{Objects Undergoing Modulations at a Known Frequency (Example: fMRI)}

The temporal variations in fMRI have a very special characteristic: they are periodic with a known frequency (the frequency of the paradigm). Consider one spatial point in an fMRI study in which $n_{p}$ on/off paradigm cycles are imaged with a time resolution of $n_{t}$ time points per paradigm cycle. The temporal frequency spectrum for this point is depicted in Fig. 7a: $n_{t}$ peaks separated by $n_{p}$ frequency points. If the signal is perfectly periodic, repeating exactly every $n_{t}$ time points, the corresponding spec-
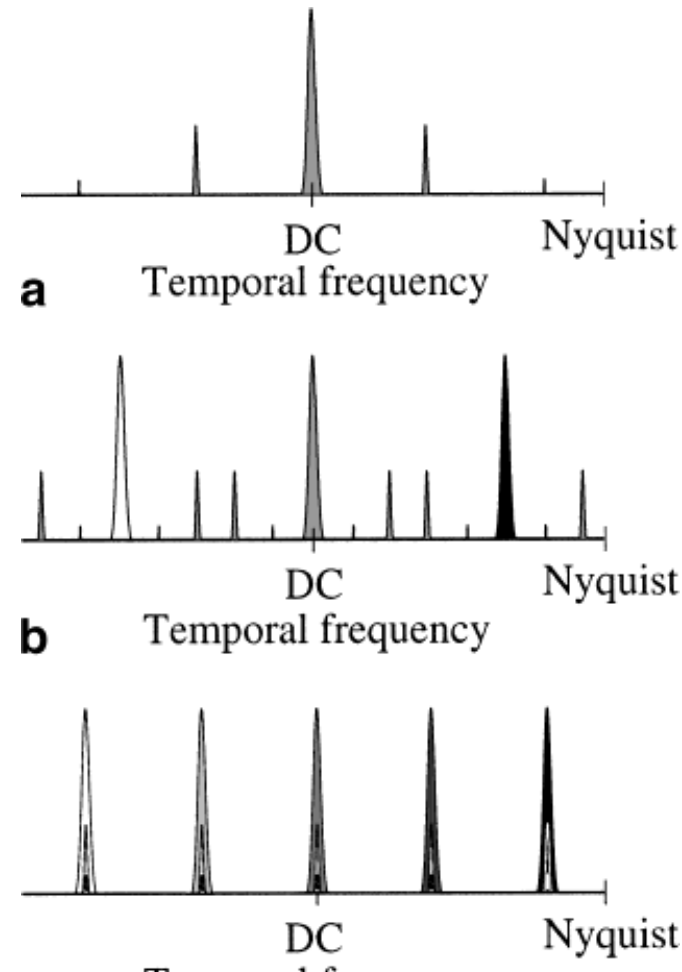

C Temporal frequency

FIG. 7. a: In an fMRI study, the time variations give rise to a spectrum expected to be non-zero only at the paradigm frequency and its harmonics. Notice the empty space between adjacent peaks. b: The present method allows a more efficient use of the bandwidth, by interleaving two or more (three in this drawing) spectra like the one shown in a. Peaks of different spectra are given different colors (white, gray, black) to facilitate visualization. c: Bad combinations of $n$ and $n_{t}$ lead to an overlap of spectra. Five spectra share the same bandwidth in $\mathbf{c}$, and a different shade of gray is associated to each one. Because each spectrum is made of five peaks (as shown in a), there are twenty-five peaks in c. However, these peaks are overlapped by groups of five, and only the central peak of each spectrum can be clearly seen in c.

trum has only $n_{t}$ delta functions separated by $1 /\left(n_{t} T\right) \mathrm{Hz}$, where $T$ is the time between images (in seconds). It is assumed here that the $n_{t}$ multiples of the fundamental frequency $1 /\left(n_{t} T\right)$ are sufficient to characterize the signal variations. If the signal is not perfectly periodic, each delta function broadens to a width related to the variation in the response during the study. Notice the empty areas between the peaks in Fig. 7a, which appear if the bandwidth of the variation in fMRI signal from cycle to cycle is narrow compared to $1 /\left(n_{t} T\right)$. Time is spent acquiring these empty areas, which contain only frequencies having no correlation with the periodic paradigm. Using the method introduced in the first part of the Theory section, two or more spectra can be forced to share the same bandwidth, as depicted in Fig. 7b. The next paragraphs explore more carefully how $n$ spectra can be interleaved in the same bandwidth [separated by $1 /(n T) \mathrm{Hz}$ ], reducing by a factor $n$ the amount of data required to achieve a given spatial and temporal resolution.

The total number of points in the temporal frequency domain is $n_{p} n_{t}$. The goal is to use these points to store as 
many spectra as possible, without overlap. As explained above, the individual peaks in a spectrum may have some width ( $\Delta$ points), and cannot in general be considered as delta functions. With $n_{p}$ points between adjacent peaks, no more than $n_{p} / \Delta$ spectra can be interleaved. The efficiency of the encoding can be maximized by choosing $n_{p}$ to be a multiple of $\Delta$, and a number of interleaved spectra $n=$ $n_{p} / \Delta$. Poor combinations of $n$ and $n_{t}$ lead to overlap of the spectra, as shown in Fig. 7c. To avoid this, the frequency difference between two spectra $\left(N_{1} /(n T)\right.$ where $N_{1}$ is an integer) must not be equal to a multiple of the paradigm frequency $\left(N_{2} /\left(n_{t} T\right)\right.$ where $N_{2}$ is an integer)

$$
\frac{N_{1}}{n} \neq \frac{N_{2}}{n_{t}}
$$

for all possible $N_{1}$ and $N_{2}$, where $N_{1}<n$ and $N_{2}<n_{t}$ (to avoid the aliasing of $N_{1} /(n T)$ and $N_{2} /\left(n_{t} T\right)$ beyond the $1 / T$ bandwidth). For example, the case $n=3$ and $n_{t}=5$ in Fig. 7b does not lead to overlap, unlike the case $n=5$ and $n_{t}=5$ in Fig. 7c where Eq. [6] is violated with $N_{1}=1$ and $N_{2}=1$. (Technically, with some extra complications, overlap like that shown in Fig. 7c can be avoided without changing $n$ or $n_{t}$, if a Cartesian imaging method is used. This involves choosing an increment for $f$ in Fig. 3a that is not $1 / n$ of a line, so that the $n$ spectra are not uniformly spaced across the bandwidth. For the present work, only the simpler case of $n$ and $n_{t}$, such that Eq. [6] is obeyed, is considered.)

In Fig. 7b, the frequency components of both the black and the white spectra are found at known locations: these spectra are centered at respectively $+1 /(n T)$ and $-1 /(n T)$ $\mathrm{Hz}$, and they have peaks every $1 /\left(n_{t} T\right) \mathrm{Hz}$. A filter can be used to remove these two spectra, and preserve only the one from nonaliased material (centered at DC in Fig. 7b). This filter has $\Delta$ zeros centered at the frequency of each peak to be removed, and is equal to one elsewhere. The higher harmonics of the spectra to be removed may contain mostly noise, with negligible signal, and one should consider whether to remove such harmonics. Indeed, filtering out frequency components filled only with noise would actually decrease our knowledge of the noise characteristics, making the determination of whether an observed effect is likely to be true activation or random fluctuations more difficult (45). While some frequency components must be zeroed to eliminate the aliasing, the number of frequency points zeroed should be kept to a minimum to optimize the significance of activated regions.

In summary, UNFOLD allows one to encode some spatial information along the $t$ axis of $k-t$ space. If all the points in an object need a full bandwidth to be adequately represented, then such a transfer of information from the $k$ to the $t$ axis is useless. However, if an object contains points which require only a fraction of the available bandwidth (like the spectra in Fig. 6b and 7a), it might be advantageous to use the free parts of the spectra to encode information about other spatial points. In the following section, the UNFOLD method is used to speed up cardiactriggered imaging as well as fMRI.

\section{RESULTS}

\section{Cardiac-Triggered Imaging}

\section{Evaluation of UNFOLD's Behavior}

A cardiac-triggered study $(1,2)$ was performed on a $1.5-\mathrm{T}$ imager (Signa Echospeed, GE Medical Systems, Milwaukee, WI). Sixteen cardiac phases were acquired; the 8th is shown in Fig. 8a. Using only the even $k_{y}$ lines for the even time frames, and the odd $k_{y}$ lines for the odd frames, 16 time frames (corrupted by aliasing) were then reconstructed. These images were used as input to the UNFOLD method, which generated a series of images where the aliasing was unwrapped back to its original position. The 8th time frame of the input and the output of UNFOLD are shown, respectively, in Fig. 8b and c. The main goal of the present section is to evaluate the behavior of UNFOLD by comparing the resulting images (reconstructed using only one half of the acquired data, e.g., Fig. 8c), to the true images (reconstructed using all the data, e.g., Fig. 8a).

An ROI labeled $A$ (height $F O V_{y} / 2$ along $y$ ) containing the heart is shown in Fig. 8a. An aggregate temporal frequency spectrum for region $A$ (the sum of the magnitude of the temporal frequency spectra at each point contained in $A$ ) is shown in Fig. 9a. The aggregate temporal frequency spectrum for region $B$ (shown in Fig. 8a) is presented in Fig. 9b. As expected, the voxels in the region containing the heart (Fig. 9a) tend to have a wider distribution of frequencies than the voxels located outside the heart (Fig. 9b). In Fig. $8 \mathrm{~b}$, the ROIs $A$ and $B$ are overlapped through aliasing. The aggregate temporal spectrum of the resulting $(A+B) \mathrm{ROI}$ is shown in Fig. 9c. The component at DC in Fig. 9c is the spectrum from region $A$ (Fig. 9a) while that at the Nyquist frequency is from the (aliased) region $B$ (Fig. 9b). Aliased and nonaliased components were separated using the Fermi filter plotted in Fig. 9c. This filter was selected (automatically) using an algorithm that separates the two peaks of a spectrum such as Fig. 9c, while providing as wide a bandwidth as possible to the central (dynamic) component. The filter in Fig. 9c was

$$
F(E)=\frac{1}{1+\exp \left(\frac{E-E_{f}}{k T}\right)},
$$

with $k T=0.022 N y$ and $E_{f}=0.79 N y$, where $N y$ is the Nyquist frequency. Figure 9 is in fact an experimental equivalent to the stylized Fig. 6 (with $B W_{1}=B W_{2}$ ). Once aliased and non-aliased components are separated using the filter, images can be generated in which the aliasing is corrected (Fig. 8c). In the "more dynamic" half of Fig. 8c, the SNR was measured to be 0.81 that of Fig. 8a (theoretical value of 0.80 from Eq. [5]), while this factor was measured to be 1.54 in the "less dynamic" area (theoretical value of 1.63).

Figure $8 \mathrm{~d}$ presents one attempt at evaluating the quality of UNFOLD's results. It is the absolute value of the difference between the magnitude images in Fig. 8a ("truth") and 8c (result), windowed to show clearly the noise level; a given shade of gray corresponds to a magnitude that is ten times lower in Fig. 8d than in 8a-c. Almost no anatomic 

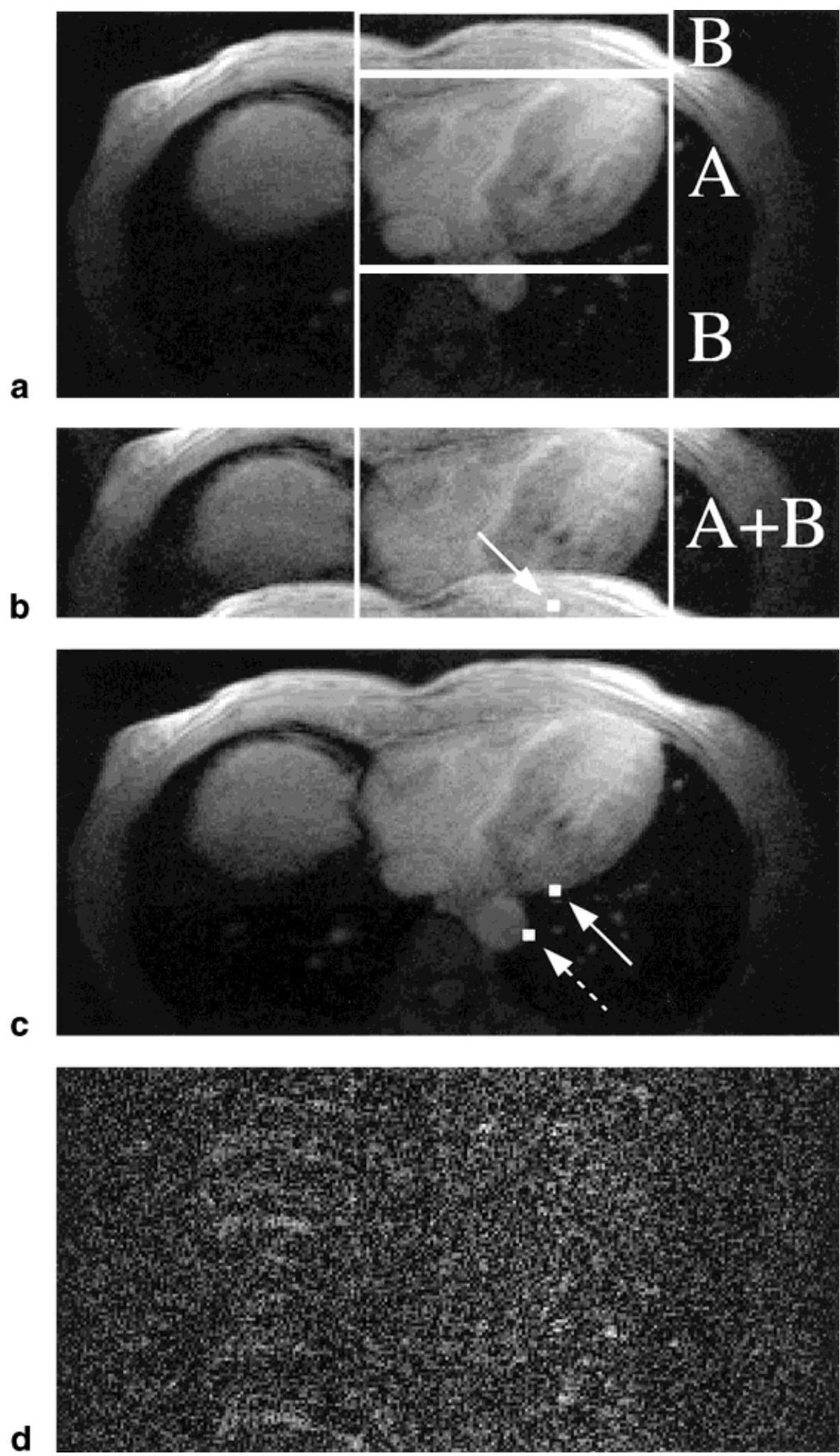

FIG. 8. Eighth phase of a 16 phase cardiac-triggered study (axial images, 2DFT gradient-echo sequence, respiratory compensation, through-slice flowcompensation, TE $=6.0 \mathrm{msec}$, slice thickness $=5 \mathrm{~mm}$, FOV $=32 \times 16 \mathrm{~cm}$, matrix size $=256 \times 128$, phase-encoding along $\mathrm{A} / \mathrm{P}$, surface coil, peripheral gating, acquired over 128 heartbeats). a: A "truth" is reconstructed in the conventional way from the acquired data. ROI's $A$ and $B$ are defined for future reference. $\mathbf{b}$ : Using half of the acquired data (even $k_{y}$ lines for even time frames and odd $k_{y}$ lines for odd time frames) a series of images corrupted by aliasing is reconstructed, and input into UNFOLD. The regions $A$ and $B$, which were distinct in $\mathbf{a}$, are overlapped because of aliasing. c: In the output of UNFOLD, the aliased component is "unfolded" back to its original position. Small ROls $(3 \times 3$ pixels) are defined for future reference (the ROls are smaller that the squares used to indicate their location). One of the two ROls is also shown in b. d: Absolute value of the difference obtained by subtracting $\mathbf{c}$ from $\mathbf{a}$. The windowing used in $\mathbf{d}$ is 10 times narrower than the windowing used in $\mathbf{a}, \mathbf{b}$, and $\mathbf{c}$. features can be recognized in the difference image, indicating that UNFOLD successfully corrected the aliasing in this frame. Figure 10 compares more quantitatively truth and treated images by showing the mean magnitude of difference images (like Fig. 8d) over the region containing the heart (ROI $A$ in Fig. 8a) normalized by the mean magnitude calculated over ROI $A$ of the truth images (the average is performed over the frame number as well as over the ROI), as a function of the cardiac phase. The 'truth' and the treated images differ by $5-6 \%$ in the region of the heart. This difference comes partly from imperfect aliasing removal, and partly from random noise. The dashed straight line in Fig. 10 shows that the level expected due to noise is around $3.8 \%$. (This value is measured from an area of the 

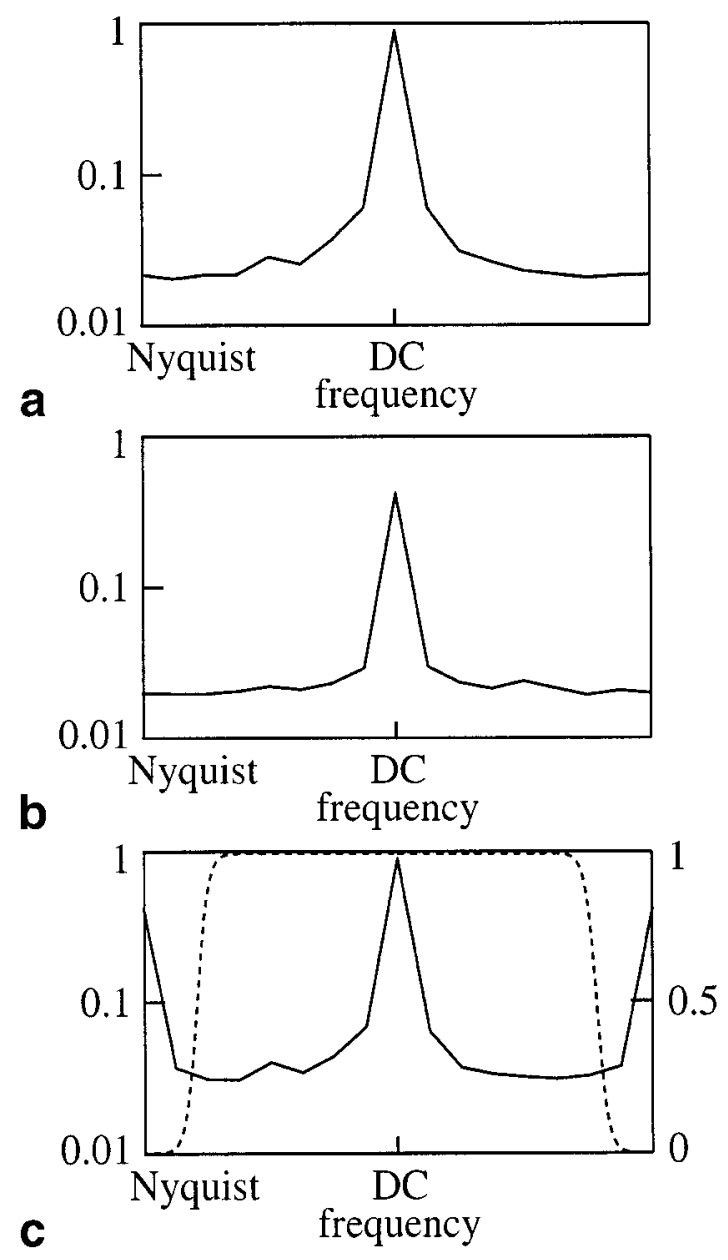

FIG. 9. a: Aggregate temporal frequency spectrum for the material found in the region $A$ containing the heart (shown in Fig. 8a). b: Aggregate temporal frequency spectrum for the material found in the region $B$ (shown in Fig. 8a). Heart material (region $A$ ) tends to have a wider frequency spectrum than the less dynamic material outside the heart (region $B$ ). c: Aggregate temporal frequency spectrum for the material found in the region $(A+B)$ (shown in Fig. 8b). Signal from regions $A$ and $B$ can be separated by using the filter plotted with the dashed line (which uses the linear vertical axis at the right of the plot).

image where no signal is present. With an SNR of 16 over the heart (as measured in the reference data set), simulations yield a similar value of $4.1 \%$.)

The time behavior of two small ROIs (shown in Fig. 8c) is given in Fig. 11 (for the full arrow in Fig. 8c) and Fig. 12 (for the dashed arrow). In both cases, the solid and the longdashed curves represent, respectively, the truth and UNFOLD's output. The short-dashed curve represents images obtained using "data sharing” (in which each time frame is generated by combining a frame made of only odd $k_{y}$ lines with a neighboring frame, made of even $k_{y}$ lines) to generate 16 nonaliased time frames from the 16 aliased frames. Both figures use the same normalization for the vertical axis. The ROI indicated by the full arrow in Fig. 8c was chosen because wide magnitude variations occur as the myocardium moves in and out of the small ROI, and because a high level of aliasing was present at this location (Fig. 8b). Notice in Fig. 11 that the solid and long-dashed curves are very similar, indicating that UNFOLD has been

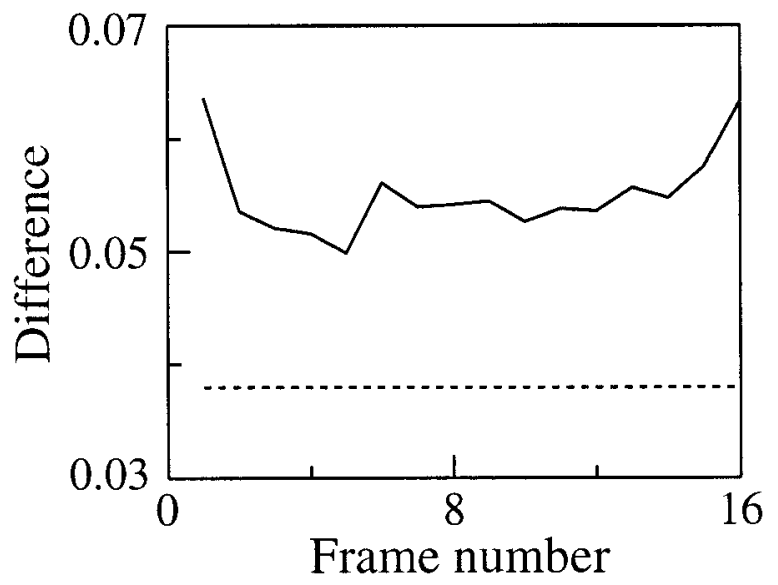

FIG. 10. Mean (over the ROI $A$ shown in Fig. 8a) of the absolute value of the difference between the magnitudes of the 'truth' and the treated images. The normalization factor is the mean (over the same $\mathrm{ROI} A$ ) of the (magnitude of the) truth, averaged over all the cardiac phases. Notice that UNFOLD performs better (smaller difference) for intermediate time frames than for the first and last ones. This problem results from a magnitude discontinuity between the last and first time frame ('lighting artifact'), and can be alleviated with postprocessing.

reasonably successful in representing the time behavior of this ROI while using only half the acquired data. By comparison, data sharing causes reduced temporal resolution. The dashed arrow ROI is adjacent to the descending aorta, and the intensity varies as the aorta moves in and out of the region. As can be seen in Fig. 8a, the aorta was in the less dynamic region $B$ and as a result, only a limited temporal bandwidth was used by UNFOLD to describe the time behavior. Accordingly, the method provides only a smoothed representation of the actual variations in the ROI (as can be seen in Fig. 12), and the aliased version of the aorta is not completely removed from region $A$ (i.e., a faint artifact is generated in the more dynamic region). In region $B$, the temporal resolution of UNFOLD is lower than that of

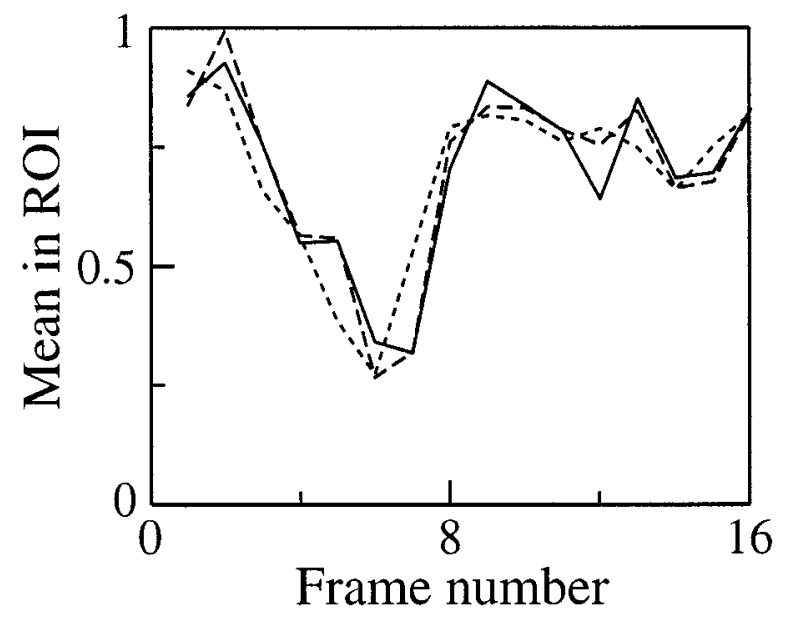

FIG. 11. Mean in the ROI indicated by the solid arrow in Fig. 8b and $c$ as a function of cardiac phase. A comparison of the representation of this ROI in the truth, the treated images and the images obtained using data sharing is made in the text. 


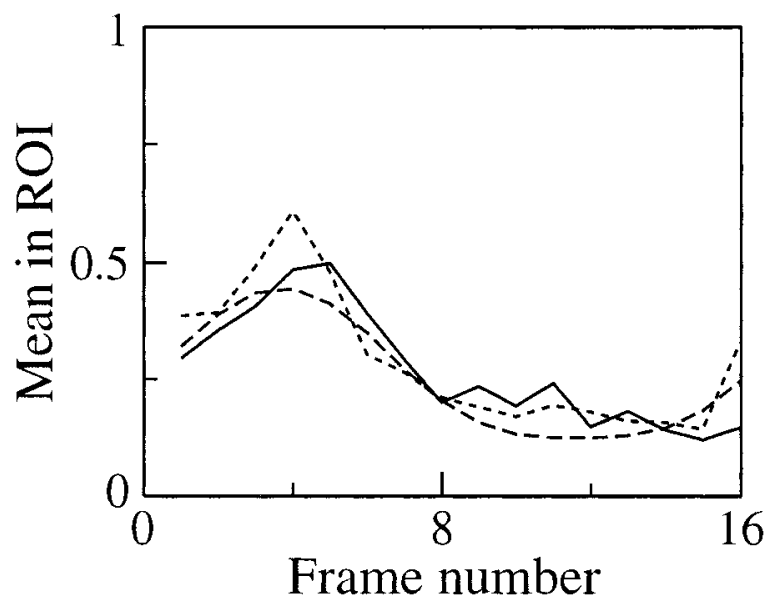

FIG. 12. Mean in the ROI indicated by the dashed arrow in Fig. 8c, as a function of cardiac phase. The vertical axis has the same normalization as in Fig. 11. This graph is used in the text to compare the truth, the treated images and the images obtained by using data sharing.

data sharing. Ideally, the aorta should be part of the dynamic region $A$ instead of the less dynamic region $B$. However, the dynamic region cannot be larger than $F O V_{y} / 2$ in the phase-encoding direction, and could not contain both the beating heart and the aorta. The solution to this problem would consist in increasing slightly $F O V_{y}$. Note that even though the assumption of UNFOLD was not fully obeyed in this case, this violation did not produce disastrous artifacts. If the true temporal bandwidth in the less dynamic region is wider than expected, only the higher temporal frequency components will spatially alias, and they will affect the higher temporal frequencies within the dynamic region.

Unlike UNFOLD, which allows half the FOV to have a higher temporal resolution than the other half, data sharing yields a uniform, intermediate temporal resolution over the whole FOV. In Fig. 11, the higher temporal resolution in the dynamic region allows UNFOLD to depict rapid variations more accurately than data sharing, while in Fig. 12 , the intermediate time resolution of data sharing provides a better representation of the truth than the low temporal resolution of UNFOLD in this region.

\section{Application of UNFOLD}

A cardiac-triggered study was performed using a segmented gradient-echo sequence (46). As required by UNFOLD, this sequence was modified such that the phase encodings for every odd time frame were shifted by half a $k_{\mathrm{y}}$ line. Eight $k_{\mathrm{y}}$ lines were acquired during each heart beat, leading to a temporal resolution of $8 T R(T R=8.6 \mathrm{msec})$ and a scan time of 16 heartbeats for $128 k_{y}$ lines. Fifteen cardiac phases were obtained during the breath-held scan. Figure 13a shows the conventional reconstruction of the 7th frame. UNFOLD expects a cyclical alternation between shifted and nonshifted sampling functions, which cannot be achieved with an odd number of cardiac phases. This problem was solved by copying the 14th cardiac phase to a (fictitious) 16th phase. After the UNFOLD processing, the 16th treated cardiac phase was discarded.
Figure 13b shows the aggregate temporal frequency spectrum calculated over the ROI shown in Fig. 13a, which covers the heart. The peak at DC contains the signal from the (non-aliased) heart, while the peak at the Nyquist frequency contains aliased signal. The spectrum in Fig. 13b was used to compute the Fermi filter (dashed line, $E_{f}=0.76$ $N_{y}$ and $k T=0.022 N_{y}$ ) in the manner mentioned previously. This filter was used to separate aliased and nonaliased signal, generating a series of 15 treated images. Figure 13c is one of these de-aliased images (7th frame) and is a corrected version of Fig. 13a. The treated images consist of a dynamic half (which was given about $76 \%$ of the temporal bandwidth by the filter in Fig. 13b) and a less-dynamic half (which used the remaining $\sim 24 \%$ of the bandwidth). The ROI of Fig. 13a (also shown in Fig. 13c) is displayed in Fig. 13d for the 15 treated time frames. Changes in the size and shape of the heart and aorta can be seen as a function of cardiac phase. Segmented cardiactriggered sequences often use data sharing to increase the number of time frames generated (47); with UNFOLD as well, the number of time frames could be further increased through time interpolation if so desired.

\section{fMRI}

\section{Evaluation of UNFOLD's Behavior}

An fMRI study was performed using a six-interleave spiral imaging sequence (43). Bilateral finger tapping was performed for 27 seconds, and then stopped for $27 \mathrm{sec}$. Six such paradigm cycles were imaged $\left(n_{p}=6\right)$. The acquired data was reconstructed in four distinct ways: a reference data set was obtained using all six spiral interleaves at each time frame, and the amount of information used in the reconstruction was diminished by a factor $n$ of 2, 3, and 6 using, respectively, only 3,2 , and 1 interleave(s) at each time frame.

As required by UNFOLD, different interleaves must be used from one time frame to the next. The acquisition and reconstruction strategy is depicted in Fig. 14 for the reference and the $n=6$ cases. The six interleaves chosen for the reference frames are indicated by straight line segments, while the interleaves used in the $n=6$ case (only one per time frame) are shown in bold. After every group of six interleaves, one interleaf is left unused to maintain a consistent time interval between time frames. Although not depicted, the strategy presented in Fig. 14 also ensures a uniform time sampling for the cases $n=2$ and 3. Clearly, discarding data degrades the SNR; the strategy was only used to provide a comparison between reference and UNFOLD results that is as fair as possible. In an actual UNFOLD application (as the one presented later) all the acquired data would be used.

Seventy-eight time frames were reconstructed from the acquired data $\left(n_{t}=13\right.$ images per paradigm cycle). Figure 15a.1 shows one of the 78 images from the reference data set. The temporal frequency spectrum for the voxel indicated by the arrow in Fig. 15a.1 is shown in Fig. 15a.2. Because the expected variations are co-periodic with the paradigm, only the frequencies indicated by tick marks should contain the activation signal. The arrows in Fig. 15a.2 point to the peaks located at the fundamental frequency of the paradigm; the presence of such peaks 


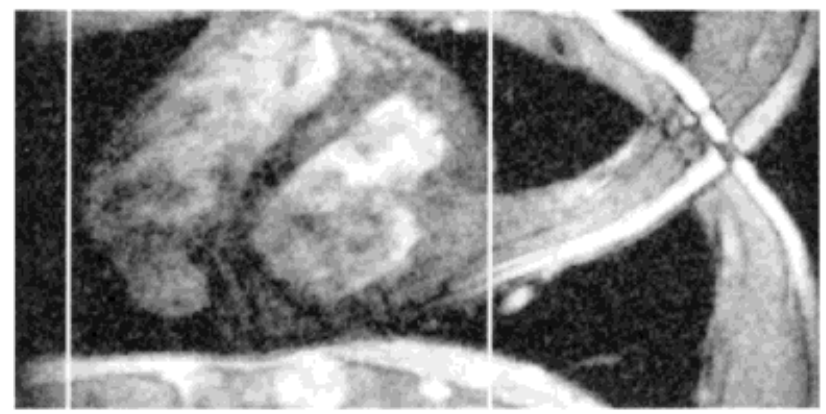

a
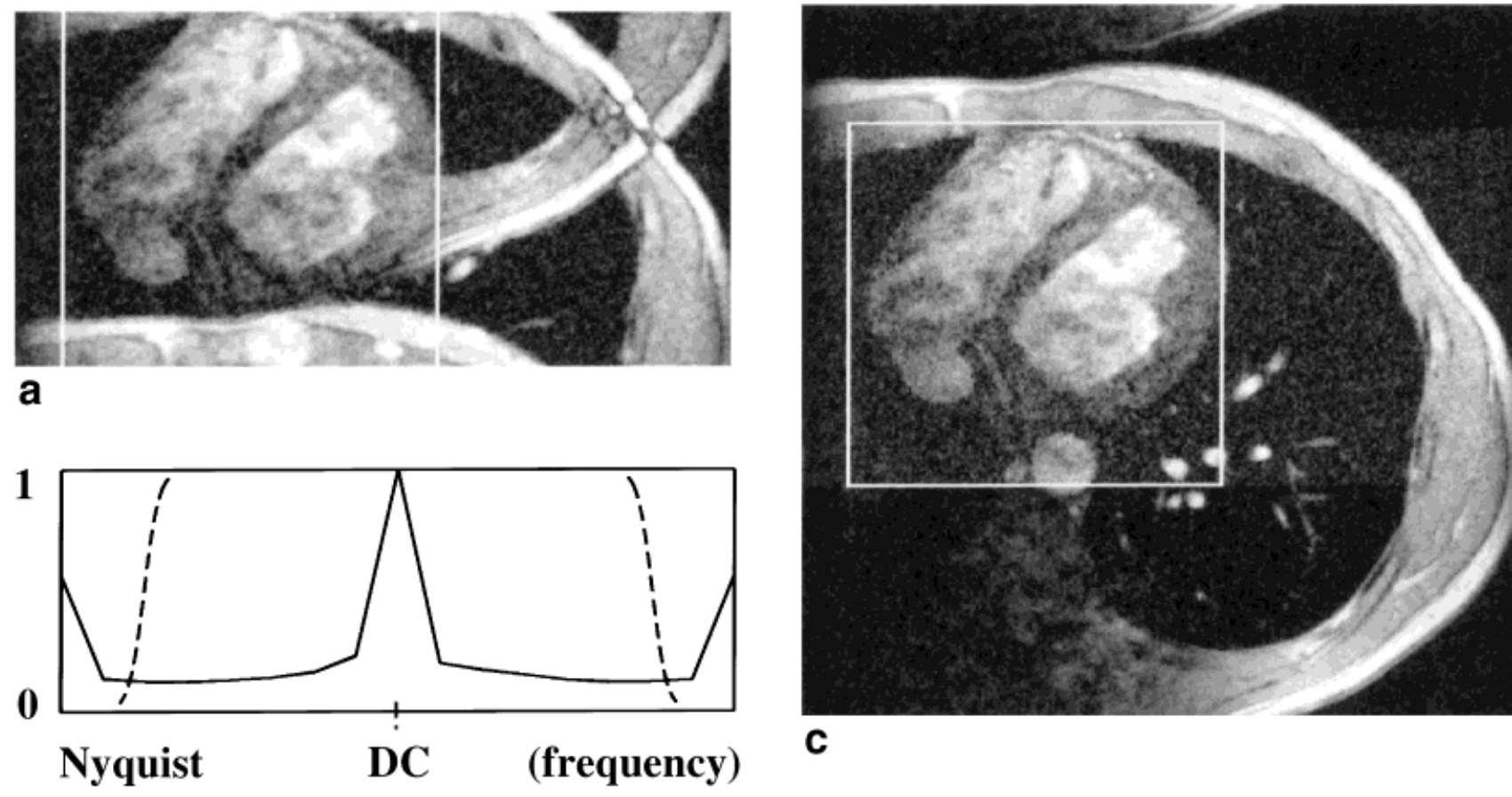

C

b
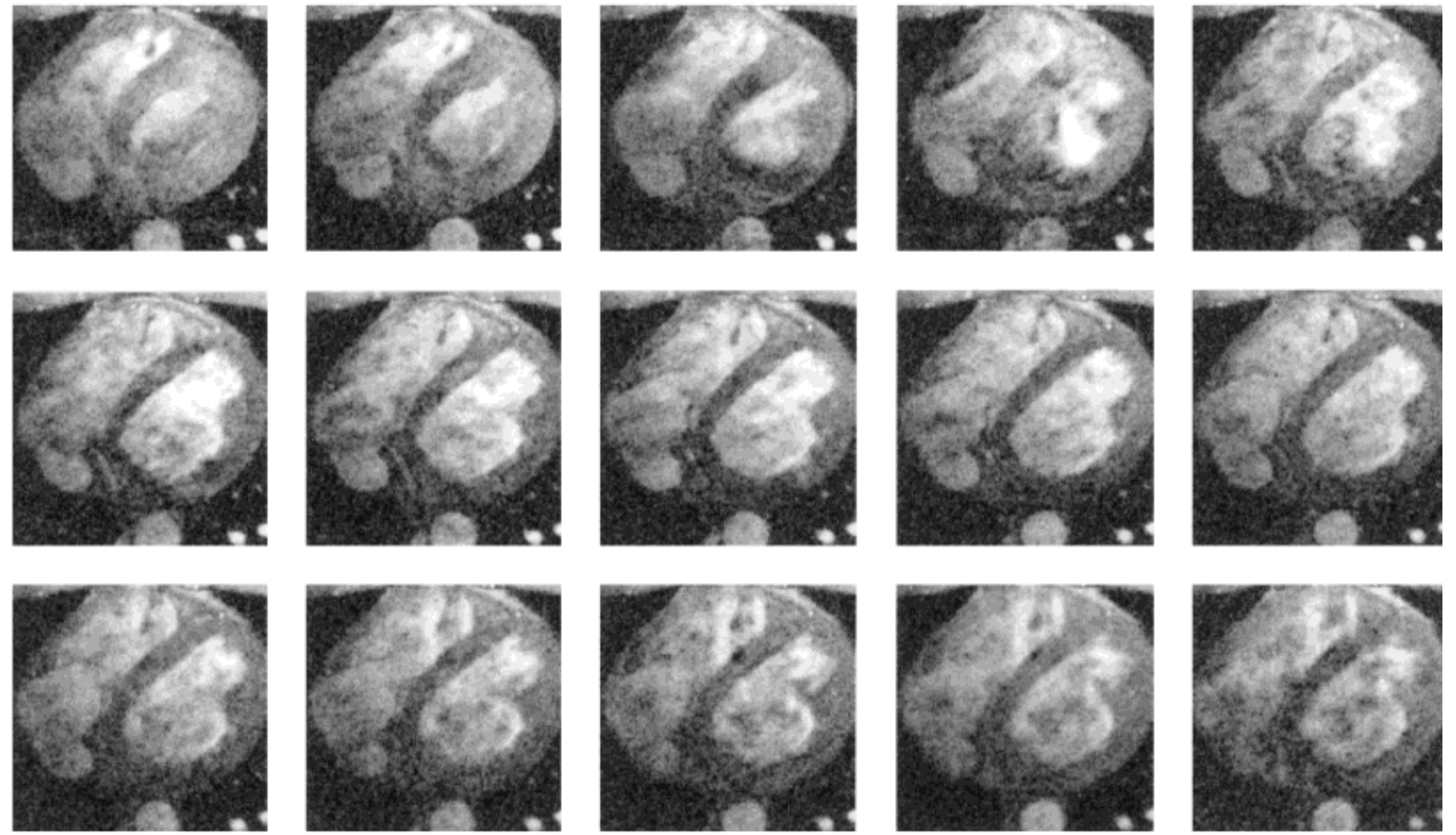

d

FIG. 13. Results obtained with a segmented cardiac-triggered gradient-echo sequence modified to shift the sampling function of every odd time frame by half a $k_{y}$ line, as required by UNFOLD $\left(T R=8.6 \mathrm{msec}, T E=3.6 \mathrm{msec}, 20^{\circ}\right.$ flip angle, 5 -mm slice thickness, $8 k_{y}$ lines per segment, $128 k_{y}$ lines, $22 \mathrm{~cm}$ by $11 \mathrm{~cm}$ FOV, 15 cardiac phases, data acquired over 16 heartbeats while the volunteer holds her breath). a: The 15 acquired images are corrupted by spatial aliasing (7th cardiac phase is shown here). b: The aggregate temporal frequency spectrum over the ROI shown in a consists of two peaks. These two peaks are separated using the Fermi filter shown with a dashed line. c: Treated images are generated where the aliasing problem is corrected (the same cardiac phase as in a is shown here). $\mathbf{d}$ : The ROI shown in a and $\mathbf{c}$ is displayed for all the 15 cardiac phases ( $3 / 4$ scale compared with $\mathbf{a}$ and $\mathbf{c})$. Order is from left to right. Windowing is the same for $\mathbf{a}, \mathbf{c}$, and $\mathbf{d}$. 
123456123456123456123456123456123456123456123

\begin{tabular}{lllllll}
$\overline{\text { frame } 1}$ & frame 2 & frame 3 & frame 4 & frame 5 & frame 6 \\
\hline
\end{tabular}

FIG. 14. This figure depicts how spiral interleaves are pooled to form time frames, in the reference and in the $n=6$ cases. See text for details. indicates that this voxel was activated by the paradigm. Figure 15a.4 is the activation map obtained from the 78 reference images. The motor cortex for both hands (including the pixel considered in Fig. 15a.2) has statistically significant correlation. Figures 15b.1, c.1, and d.1 show what happens to Fig. 15a.1 as the number of spiral interleaves used in the reconstruction is diminished by two, three, and six, respectively. The images are corrupted by aliasing of increasing severity. Figures 15b.2, c.2, and d.2 show the effect of UNFOLD on the temporal frequency spectrum of the voxel highlighted in Fig. 15a.1. The spectrum of Fig. 15b.2 has two components. One is the spectrum of the desired voxel, centered at DC. The second, centered at the Nyquist frequency, contains the contribution from object locations which are aliased onto this same image point. In contrast to the case in FT encoding, this
.1

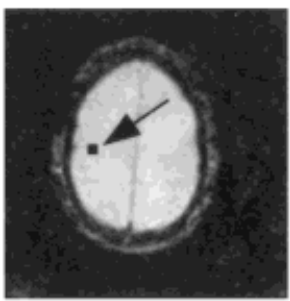

b

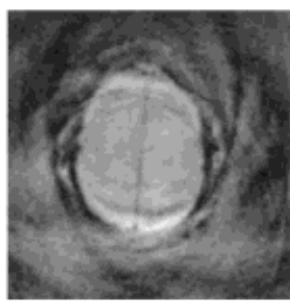

$\mathbf{C}$

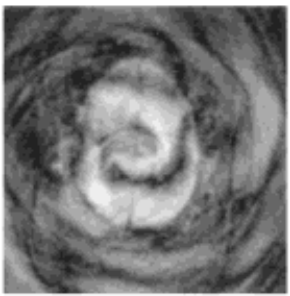

d

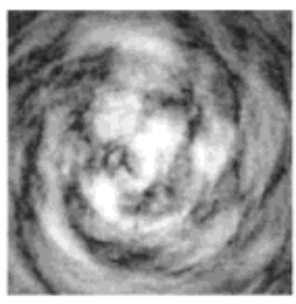

.2
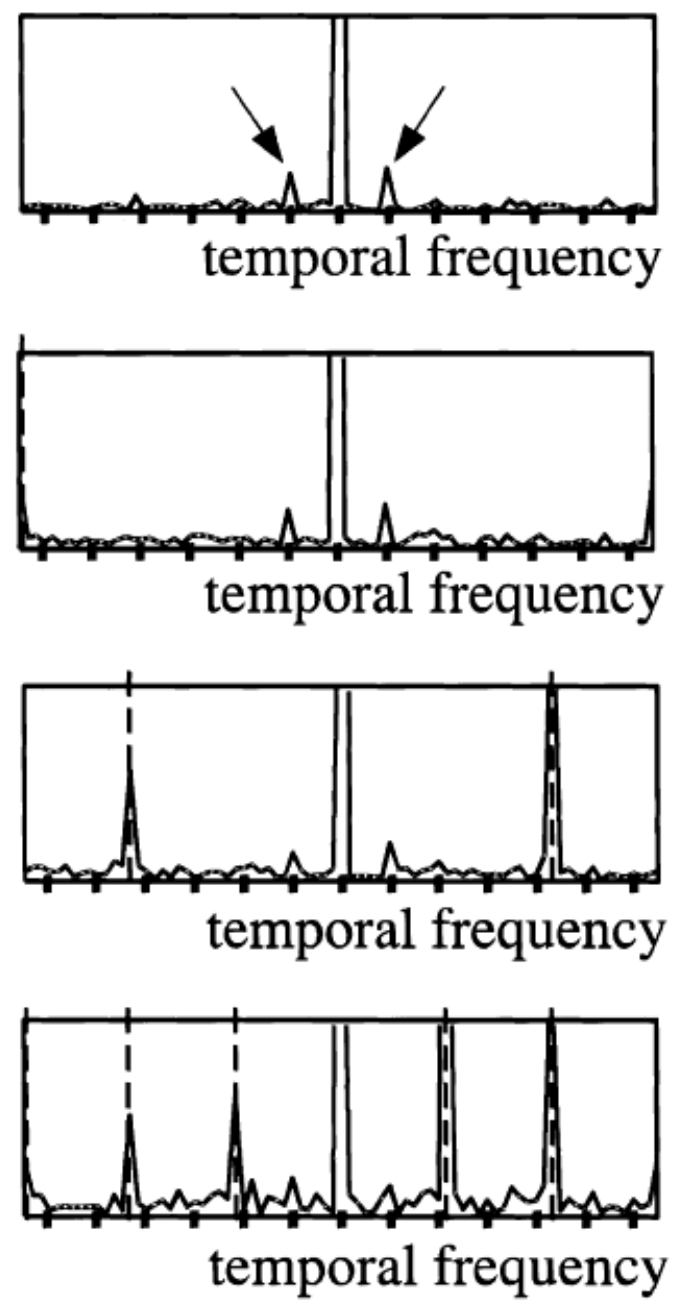

.3

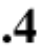

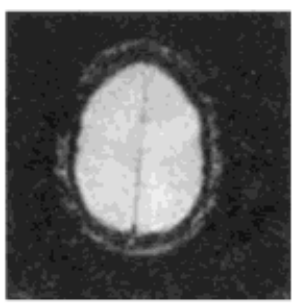
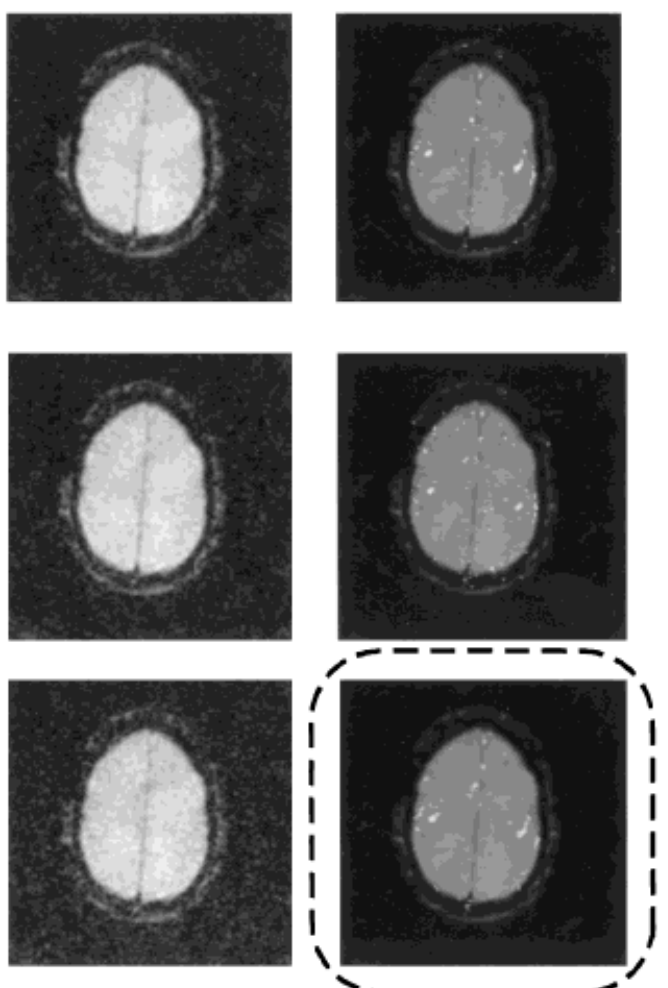

FIG. 15. Results coming from a bilateral finger tapping fMRI study [78 time frames, each one made of 6 spiral interleaves, axial images, matrix size $128 \times 128,54 \mathrm{sec}$ paradigm cycles ( $27 \mathrm{sec}$ on followed by $27 \mathrm{sec}$ off), 13 images per paradigm cycle, 10 slices, TR $=600 \mathrm{msec}$ (10 times $60 \mathrm{msec}$ ), TE $=40 \mathrm{msec}, 4-\mathrm{mm}$ slice thickness, $24 \mathrm{~cm} \mathrm{FOV].} \mathbf{a}, \mathbf{b}, \mathbf{c}$, and $\mathbf{d}$ represent, respectively, the truth (where six spiral interleaves are used to reconstruct each time frame) and cases where the number of interleaves per frame is reduced by a factor $n$ of 2,3 , and 6 . Images in column . 1 show one of the 78 time frames acquired. Column .2 gives the temporal frequency spectrum for the voxel whose location is shown in image a.1 (the square in a.1 is bigger than the voxel). Ticks on the horizontal axis indicate the frequency values where activation signal is expected, and the dashed lines indicate the frequency components to be zeroed. Column .3 shows the same images as column .1, after they are treated with UNFOLD. Column .4 gives the activation maps obtained from the images shown in column .3 (overlaid onto the time average). Unlike b.4 and c.4, d.4 provides an SNR-equivalent comparison with a.4 (see text for details). Notice that the aliasing artifacts in images b.1, c.1, and d.1 are removed in images b.3, c.3, and d.3, and that the activated motor cortex in a.4 can also be seen in b.4, c.4, and d.4. This suggests that UNFOLD can remove the aliasing artifacts in fMRI while preserving most of the activation signal. 
aliasing does not come from a single point in the object. However, assuming a proper choice of $n$ and $n_{t}$ was made (Eq. [6]), it is guaranteed to be zero (except for noise) at DC, the fundamental activation frequency and its harmonics. The aliasing can be removed by zeroing all the harmonics of the spectrum centered at the Nyquist frequency; however, zeroing only the zeroth order peak with a width $\Delta$ equal to one (only the point at the Nyquist frequency was zeroed) proved sufficient. The result is shown in Fig. 15b.3, where the aliasing (clearly visible in Fig. 15b.1) has been removed. The activation map of Fig. 15b.4 (cf. Fig. 15a.4) was obtained from the data set represented in Fig. 15b.3.

Similarly, reducing the number of interleaves per time frame by a factor $n$ of 3 and 6 forces $n$ spectra to share the same bandwidth (Fig. 15c.2 and d.2). By zeroing one frequency point every $1 /(n T) \mathrm{Hz}$ (as indicated by dashed vertical lines), images like Fig. 15c.3 and d.3 were obtained. The activation map of Fig. 15c.4 was obtained from the data set in Fig. 15c.1. Notice that Fig. 15b.3, c.3, and d.3 are obtained using, respectively, two, three, and six times less data than Fig. 15a.1. Accordingly, the activated regions progressively decrease in statistical significance. The following strategy was used to provide an SNR-equivalent comparison between reference and UNFOLD results. For each value of $n$, there are $n$ independent ways to choose one $n$th of the interleaves. These were processed separately to yield $n$ sets of images like Fig. 15b.3, c.3, or d.3. These $n$ sets were then averaged before performing the correlation process. This is appropriate in a demonstration of the method's behavior, but would not be possible in practice. An example of the correlation maps obtained from averaged data sets is shown in Fig. 15d.4 for the case $n=6$ (cf. Fig. 15a.4). The dashed line around Fig. 15d.4 emphasizes that unlike the other images in column .4, d.4 is obtained from an averaged data set (and therefore has better SNR than b.4 or c.4). Note that any aliasing surviving the filtering process would disappear through such averaging; however, Fig. 15d.3 shows qualitatively that UNFOLD worked well in removing the aliasing. Fig. 15d.4 is very similar to the reference activation map a.4 (SNR-equivalent comparison). Results nearly identical to both Fig. 15a.4 and d. 4 have also been obtained for the cases $n=2$ and 3 (not shown here). While column .3 in Fig. 15 shows that UNFOLD removed the aliased components, the comparisons of Fig. 15a.4 with b.4, c.4, and d.4 show that activation information is retained in the processed data sets.

\section{Application of UNFOLD}

An fMRI study was performed using a 3D "stack of spirals", $(48,49)$ imaging sequence with four spiral interleaves in the $k_{x}-k_{y}$ plane, sixteen $k_{z}$ phase encodes, and $T R=250 \mathrm{msec}$ (acquisition time of $16 \mathrm{sec} /$ time frame). Finger tapping was performed while a 2 sec audio cue was on, and then stopped for $12 \mathrm{sec}$ for a paradigm period of $14 \mathrm{sec}$. Forty paradigm cycles were imaged, for a total imaging time of 9 min 20 sec. Studies using a paradigm with such a short activation period $(50,51)$ are generally referred to as singleevent or single-trial fMRI and have a smaller signal than the more conventional boxcar fMRI (52).

Note that the imaging time for a single time frame (16 $\mathrm{sec}$ ) is longer than a paradigm cycle (14 sec). Convention- ally, such a situation would make little sense, as one must resolve the time variations occurring within a paradigm cycle to generate activation maps. However, UNFOLD was used to generate (aliased) time frames using only two of sixteen $k_{z}$ values. As a result, a time frame could be generated in 2 sec, providing seven time frames per paradigm cycle $\left(n_{t}=7\right)$. All four spiral interleaves for a given $k_{z}$ phase-encode value were acquired before changing $k_{z}$. The $k_{z}$ acquisition order was modified in the following way. Let $k_{z}$ be an integer from 1 to 16 . The first time frame used $k_{z}$ of 1 and 9 , the second frame used 2 and 10 , up to the eighth time frame, which used 8 and 16. This scheme was repeated with a period of eight frames. Conventional reconstructions (e.g., Fig. 16a) are corrupted by an eight-fold aliasing in the slice direction. (Note that with only two phase-encodes per time frame, the 'arbitrary trajectory' description in the Theory section might be more appropriate than the simpler 'Cartesian grid' description). The temporal frequency spectrum for the voxel highlighted in Fig. 16a is shown in Fig. 16b. Eight spectra are interleaved, but only signal from the non-aliased material is expected at the frequencies indicated by tick marks. The presence of peaks at the fundamental frequency of the paradigm (arrows in Fig. 16b) indicates that the voxel highlighted in Fig. 16a contains activation. All the frequency terms (zeroth term, fundamentals, and harmonics) corresponding to the spectra generated by aliased components were zeroed, using a width $\Delta$ equal to five frequency points per term to be erased. This filtering process generated images like Fig. 16c, in which the aliasing seen in Fig. $16 \mathrm{a}$ has been removed. The correlation maps for four slices, overlaid onto fast spin-echo (FSE) images, are shown in Fig. 16d through g. Motor cortex activation is seen in Fig. $16 \mathrm{~d}$ and e, while auditory activation (caused by the audio cue) is seen in Fig. $16 \mathrm{f}$ and g. A simple correlation with a sinusoidal function was used; correlation with an hemodynamic response function might possibly have improved the activation maps.

\section{DISCUSSION}

UNFOLD can enable a reduction in acquisition time for individual temporal frames in a dynamic study. While UNFOLD was implemented for cardiac-triggered imaging and for fMRI, it is not applicable to all dynamic studies. For UNFOLD to be useful, it first has to be compatible with the imaging sequence used. Not all imaging sequences can support UNFOLD: a notable example is retrospectively interpolated cine $(3,4)$ (the temporal interpolation appears to be incompatible with the time-varying sampling function inherent in UNFOLD). Furthermore, for UNFOLD to be useful in a given application, the geometry or time behavior of the imaged anatomy has to meet some criteria. Indeed, there are two important assumptions inherent to the UNFOLD method. It is assumed that more than one spatial point can share the same temporal bandwidth without overlap. Second, it is assumed that enough is known about the shape of the temporal spectra to separate the contributions from these points. Failures of the UNFOLD method are caused by violation of one or both of these assumptions. Such violations give rise to a different kind of aliasing, where some temporal frequencies at a 


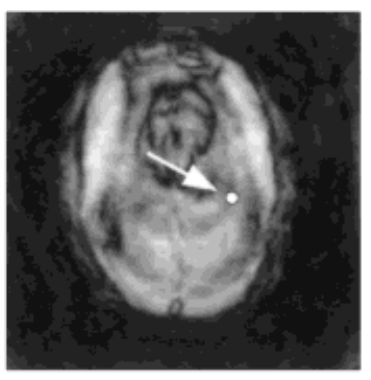

a

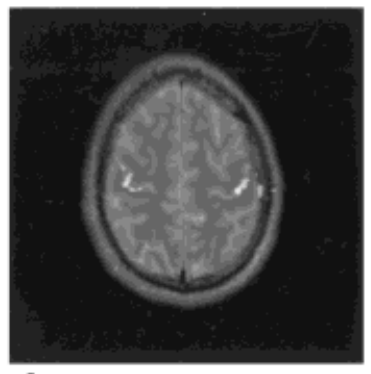

d
0.1

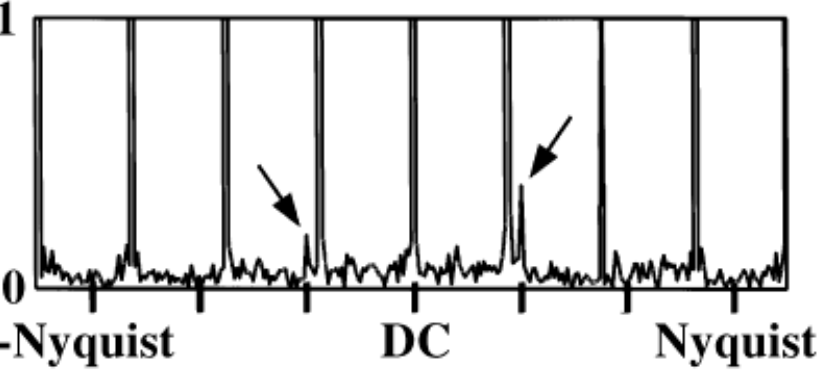

b

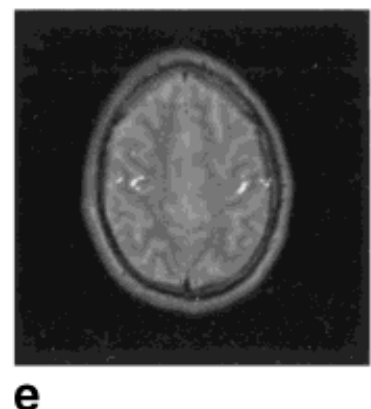

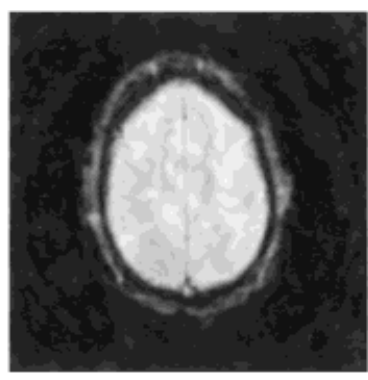

C

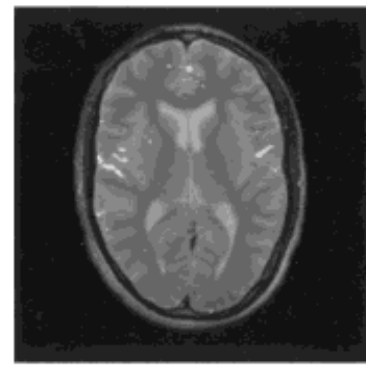

g

FIG. 16. Results obtained for a single-trial fMRI experiment (4 spiral interleaves, $16 k_{z}$ phase-encode values, axial images, matrix size $128 \times 128, \mathrm{TR}=250 \mathrm{msec}$, TE $=40 \mathrm{msec}, 5 \mathrm{~mm}$ resolution along $z, 24 \mathrm{~cm}$ FOV). Bilateral finger tapping was performed while a 2 sec audio cue was on, and then stopped for $12 \mathrm{sec}$. The acquisition time for a time frame (16 sec) is longer than a paradigm cycle (14 sec). UNFOLD is used to reduce the acquisition time by a factor 8, providing 7 frames per paradigm cycle. a: The acquired frames are corrupted by an 8-fold aliasing in the through slice direction. b: Temporal frequency spectrum for the highlighted image point in a. UNFOLD interleaves 8 spectra into the same temporal bandwidth. Marks are placed on the axis at the locations of the DC, fundamental and harmonic frequencies for the non-aliased material. Selecting only these frequencies, the aliasing seen in a is removed in c. The data set represented in $\mathbf{c}$ is used to generate the correlation map in $\mathbf{d}$ (which is overlaid onto a corresponding FSE anatomical image). e, $\mathbf{f}$, and $\mathbf{g}$ show the correlation maps for three other slices. $\mathbf{d}$ and $\mathbf{e}$ (slices 5 and 6 out of 16) show motor cortex activation, whereas $\mathbf{f}$ and $\mathbf{g}$ (slices 13 and 14) show the auditory cortex activation caused by the audio cue.

given spatial point are falsely attributed to another spatial point. On the other hand, if these assumptions are relatively robust for a given application. UNFOLD can depict accurately the time-varying object while requiring only a fraction of the data that would conventionally be needed. In cardiac imaging and fMRI, these assumptions take the form of seemingly reasonable statements: It should be possible to describe the quasi-static material outside the heart using only a limited bandwidth, and in fMRI, activation signal should be found only at temporal frequencies related to the paradigm frequency. While UNFOLD is expected to be directly applicable to myocardial perfusion studies, it remains to be seen whether it will prove useful in contrast enhanced studies of other parts of the body.

The following illustrates a problem that was encountered with the cardiac results presented in Fig. 8. Because of $\mathrm{T}_{1}$ relaxation and eddy currents, the signal magnitude in the first time frame was $10-20 \%$ higher than in the last frame ('lighting flash artifact'), violating UNFOLD's assumption that the signal in the thoracic wall varied slowly as a function of time (because it belongs to region $B$; see Fig. 8a). As a result, in the processed images, a few time frames are required to recover from the sudden changes. Although the difference images between 'truth' and UNFOLD's output showed almost no anatomic features for intermediate time frames (e.g., Fig. 8d), this was not true for the first and last time frames. This problem can be alleviated during the processing if the time series is expanded by appending all the frames except the first and last in reverse order, after the last frame. With $N$ being the number of collected frames, the ordering of the resulting $2 N-2$ frames is 1 , $2, \ldots, N-1, N, N-1, \ldots, 2$. This new time series has no discontinuity, and the need for an alternation of $k$-space sampling patterns between adjacent frames is respected. The cardiac data set presented in Fig. 13 was not affected by a lighting flash artifact, owing to the use of continuous $\mathrm{RF}$ (dummy excitations to preserve the steady state).

A conventional segmented cardiac-triggered sequence would not have been able to obtain the same spatial and temporal resolution over the heart in the same imaging time as for the data set presented in Fig. 13. To maintain the spatial resolution, twice as many $k_{y}$ lines would be required to avoid spatial aliasing, thereby doubling the scan time and making breath-held scanning more challenging. To maintain the data acquisition time, the number of $k_{y}$ lines per segment would have to be increased from 8 to 16 , reducing the temporal resolution to $16 T R$ instead of $\sim 10.5 T R$ in the dynamic region $(10.5=8 / 0.76$; the $76 \%$ is the full-width-at-half-maximum of the filter). A scan using $16 k_{y}$ lines per segment would acquire the same amount of data as the study displayed in Fig. 13; however, this data would provide uniform temporal resolution over the FOV. While this uniform temporal resolution is higher than the resolution provided by UNFOLD over the "less dynamic" 
region, it is lower for the dynamic region containing the heart (where better temporal resolution might be needed).

By increasing the temporal resolution, UNFOLD can significantly increase the activation signal in fMRI applications where the acquisition time for a time frame is of the same order as the paradigm cycle period, like the one presented. In such a case, conventional fMRI provides only negligible signal in the activation images. UNFOLD can be seen as a way of enabling certain fMRI studies that would otherwise not be possible due to an acquisition time too long for the paradigm period. It might also be used to study hemodynamic responses with an increased temporal resolution.

UNFOLD reduces the data requirements for acquisition of a temporal frame. This reduction can be converted into decreased total imaging time, better spatial or temporal resolution, or larger spatial coverage. It can also allow the use of a slower pulse sequence; for example, an investigator without access to EPI or spiral imaging could achieve adequate time resolution in fMRI with a normal 2DFT gradient-echo sequence. Alternatively, a longer TR could be used to increase the blood oxygenation level-dependent (BOLD) SNR. The use of UNFOLD to increase the spatial resolution, to increase the number of slices, or to decrease the total imaging time is accompanied by a decrease in SNR. However, use of UNFOLD to increase the spatial coverage of a 3D scan, the in-plane FOV, or the time resolution does not have an adverse effect on SNR (increasing the time resolution would reduce the SNR of the individual frames, but the SNR can be regained through a correlation process (in fMRI), or by averaging frames if necessary).

\section{CONCLUSION}

UNFOLD uses the $t$ axis of $k-t$ space to resolve information normally encoded along the $k$ axes. In some dynamic applications in which the time axis is not efficiently exploited by conventional encoding, such a reorganization of $k-t$ space can lead to a significant decrease in acquisition time for the temporal frames. As shown, this can lead to an increase in temporal resolution by nearly a factor of two in cardiac-triggered imaging, and by as much as a factor of eight in fMRI (making it possible to image activation even when the acquisition time of a single time frame is actually longer than the paradigm cycle period). Depending on the situation, this reduction in the acquisition time of a temporal frame can be translated into a reduction of the total imaging time, into an improvement of the spatial or temporal resolution, or into an increase in the spatial coverage. The reduction can also be used to allow a faster pulse sequence to be replaced by a slower one while preserving the time resolution.

\section{ACKNOWLEDGMENTS}

Supported in part by an NSERC postdoctoral fellowship grant to Bruno Madore, National Institutes of Health grant P41 RR09784, GE Medical Systems, and the Lucas Foundation.

\section{REFERENCES}

1. Lanzer P, Botvinick EH, Schiller NB, Crooks LE, Arakawa M, Kaufman L, Davis PL, Herfkens R, Higgins CB. Cardiac imaging using gated magnetic resonance. Radiology 1984;150:121-127.

2. O'Dell WG, Moore CC, Hunter WC, Zerhouni EA, McVeigh ER. Threedimensional myocardial deformations: calculation with displacement field fitting to tagged MR images. Radiology 1995;195:829-835.

3. Lenz GW, Haacke EM, White RD. Retrospective cardiac gating: a review of technical aspects and future directions. Magn Reson Imaging 1989;7: $445-455$.

4. Utz JA, Herfkens RJ, Heinsimer JA, Bashore T, Califf R, Glover G, Pelc N, Shimakawa A. Cine MR determination of left ventricular ejection fraction. Am J Roentgenol 1987;148:839-843.

5. Boxt LM. How to perform cardiac MR imaging. Magn Reson Imaging Clin North Am 1996;4:191-216.

6. Yu KK, Saeed M, Wendland MF, Derugin N, Cavagna FM, Higgins CB. Real-time dynamics of an extravascular magnetic resonance contrast medium in acutely infarcted myocardium using inversion recovery and gradient-recalled echo-planar imaging. Invest Radiol 1992;27:927-934.

7. Ogawa S, Lee TM, Kay AR, Tank DW. Brain magnetic resonance imaging with contrast dependent on blood oxygenation. Proc Natl Acad Sci USA 1990;87:9868-9872.

8. Ogawa S, Lee TM, Barrere B. The sensitivity of magnetic resonance image signals of a rat brain to changes in the cerebral venous blood oxygenation. Magn Reson Med 1993;29:205-210.

9. Yang Y, Glover GH, van Gelderen P, Mattay VS, Santha AK, Sexton RH, Ramsey NF, Moonen CT, Weinberger DR, Frank JA, Duyn JH. Fast 3D functional magnetic resonance imaging at $1.5 \mathrm{~T}$ with spiral acquisition. Magn Reson Med 1996;36:620-626.

10. Korosec FR, Frayne R, Grist TM, Mistretta CA. Time-resolved contrastenhanced 3D MR angiography. Magn Reson Med 1996;36:345-351.

11. Kaiser WA, Zeitler E. MR imaging of the breast: fast imaging sequences with and without Gd-DTPA. preliminary observations. Radiology 1989; 170:681-686.

12. Mussurakis S, Buckley DL, Drew PJ, Fox JN, Carleton PJ, Turnbull LW, Horsman A. Dynamic MR imaging of the breast combined with analysis of contrast agent kinetics in the differentiation of primary breast tumours. Clin Radiol 1997;52:516-526.

13. Choyke PL, Frank JA, Girton ME, Inscoe SW, Carvlin MJ, Black JL, Austin HA, Dwyer AJ. Dynamic Gd-DTPA-enhanced MR imaging of the kidney: experimental results. Radiology 1989;170:713-720.

14. Fransen R, Muller H-J, Boer WH, Nicolay K, Koomans HA. Contrastenhanced dynamic magnetic resonance imaging of the rat kidney. J Am Soc Nephrol 1996;7:424-430.

15. Rosen BR, Belliveau JW, Vevea JM, Brady TJ. Perfusion imaging with NMR contrast agents. Magn Reson Med 1990;14:249-265.

16. Xiang QS, Henkelman RM. K-space description for MR imaging of dynamic objects. Magn Reson Med 1993;29:422-428.

17. van Vaals JJ, Brummer ME, Dixon TW, Tuithof HH, Engels H, Nelson RC, Gerety BM, Chezmar JL, den Boer JA. "Keyhole” method for accelerating imaging of contrast agent uptake. J Magn Reson Imaging 1993;3:671675 .

18. Jones RA, Haraldseth O, Müller TB, Rinck PA, Øksendal. K-space substitution: a novel dynamic imaging technique. Magn Reson Med 1993;29:830-834.

19. Bishop JE, Santyr GE, Kelcz F, Plewes DB. Limitations of the keyhole technique for quantitative dynamic contrast-enhanced breast MRI. J Magn Reson Imaging 1997;7:716-723.

20. Chenevert TL, Helvie MA, Aisen AM, Francis IR, Adler DD, Roubidoux MA, Londy FJ. Dynamic three-dimensional imaging with partial $k$ space sampling: initial application for gadolinium-enhanced rate characterization of breast lesions. Radiology 1995;196:135-142.

21. Webb AG, Liang Z-P, Magin RL, Lauterbur PC. Application of reducedencoding MR imaging with generalized-series reconstruction (RIGR). J Magn Reson Imaging 1993;3:925-928.

22. Chandra S, Liang Z-P, Webb A, Lee H, Morris D, Lauterbur PC. Application of reduced-encoding imaging with generalized-series reconstruction (RIGR) in dynamic MR imaging. J Magn Reson Imaging 1996;6:783-797.

23. Hanson JM, Liang Z-P, Wiener EC, Lauterbur PC. Fast dynamic imaging using two reference images. Magn Reson Med 1996;36:172-175.

24. Riederer SJ, Tasciyan T, Farzaneh F, Lee JN, Wright RC, Herfkens RJ. MR fluoroscopy: technical feasibility. Magn Reson Med 1988;8:1-15.

25. Parrish T, Hu X. Continuous update with random encoding (CURE): a new strategy for dynamic imaging. Magn Reson Med 1995;33:326-336. 
26. Rasche V, de Boer RW, Holz D, Proksa R. Continuous radial data acquisition for dynamic MRI. Magn Reson Med 1995;34:754-761.

27. Spielman DM, Pauly JM, Meyer CH. Magnetic resonance fluoroscopy using spirals with variable sampling densities. Magn Reson Med 1995;34:388-394.

28. Kerr AB, Pauly JM, Hu BS, Li KC, Hardy CJ, Meyer CH, Macovski A, Nishimura DG. Real-time interactive MRI on a conventional scanner. Magn Reson Med 1997;38:355-367.

29. Du YP, Parker DL, Davis WL, Cao G. Reduction of partial-volume artifacts with zero-filled interpolation in three-dimensional MR angiography. J Magn Reson Imaging 1994;4:733-741.

30. Fredrickson JO, Pelc NJ. Temporal resolution improvement in dynamic imaging. Magn Reson Med 1996;35:621-625.

31. Hu X, Parrish T. Reduction of field of view for dynamic imaging. Magn Reson Med 1994;31:691-694.

32. Scheffler K, Hennig J. Reduced circular field-of-view imaging. Magn Reson Med 1998;40:474-480.

33. Kyriakos WE, Panych LP, Zientara GP, Jolesz FA. Implementation of a reduced field-of-view method for dynamic MR imaging using navigator echoes. J Magn Reson Imaging 1997;7:376-381.

34. Zientara GP, Panych LP, Jolesz FA. Dynamically adaptive MRI with encoding by singular value decomposition. Magn Reson Med 1994;32: 268-274.

35. Panych LP, Oesterle C, Zientara GP, Hennig J. Implementation of a fast gradient-echo SVD encoding technique for dynamic imaging. Magn Reson Med 1996;35:554-562.

36. Panych LP, Jolesz FA. Implementation of a fast gradient-echo SVD encoding technique for dynamic imaging. Magn Reson Med 1994;32: 738-748.

37. Peters RD, Wood ML. Multilevel wavelet-transform encoding in MRI. J Magn Reson Imaging 1996;6:529-540.

38. Pusey E, Yoon C, Anselmo ML, Lufkin RB. Aliasing artifacts in MR imaging. Comput Med Imaging Graph 1988;12:219-224.

39. Sprawls P. Spatial characteristics of the MR image. In: Stark DD, Bradley WG, editors. St. Louis, MO: Mosby, 1988. p 28.
40. Bracewell RN. The Fourier transform and its application. New York: McGraw Hill; 1965. p 104-107.

41. Goldfarb JW, Schmitt F, Fischer H, Haacke EM, Duerk JL. A method to remove ghosting from parametric phase distortions in EPI. In: Proceedings of the 3rd Annual Meeting of ISMRM, Nice, 1995. p. 759.

42. Buonocore MH, Gao L. Ghost artifact reduction for echo planar imaging using image phase correction. Magn Reson Med 1997;38:89-100.

43. Meyer CH, Hu BS, Nishimura DG, Macovski A. Fast spiral coronary artery imaging. Magn Reson Med 1992;28:202-213.

44. Macovski A. Noise in MRI. Magn Reson Med 1996;36:494-497.

45. Buonocore $\mathrm{MH}$, Maddock RJ. Noise suppression digital filter for functional magnetic resonance imaging based on image reference data. Magn Reson Med 1997;38:456-469.

46. Atkinson DJ, Edelman RR. Cineangiography of the heart in a single breath hold with a segmented turboFLASH sequence. Radiology 1991; 178:357-360

47. Foo TKF, Bernstein MA, Aisen AM, Hernandez RJ, Collick BD, Bernstein T. Improved ejection fraction and flow velocity estimates with use of view sharing and uniform repetition time excitation with fast cardiac techniques. Radiology 1995;195:471-478.

48. Irrazabal P, Nishimura DG. Fast three dimensional magnetic resonance imaging. Magn Reson Med 1995;33:656-662.

49. Lai S, Glover GH. Three-dimensional spiral fMRI technique: a comparison with 2D spiral acquisition. Magn Reson Med 1998;39:68-78.

50. Blamire AM, Ogawa S, Kamil U, Rothman D, McCarthy G, Ellermann JM, Hyder F, Rattner Z, Shulman RG. Dynamic mapping of the human visual cortex by high-speed magnetic resonance imaging. Proc Natl Acad Sci USA 1992;89:11069-11073.

51. Buckner RL, Bandettini PA, O’Craven KM, Savoy RL, Petersen SE, Raichle ME, Rosen BR. Detection of cortical activation during average single trials of a cognitive task using functional magnetic resonance imaging. Proc Natl Acad Sci USA 1996;93:14878-14883.

52. Bandettini PA, Cox RW. Contrast in single trial fMRI: interstimulus interval dependency and comparison with blocked strategies. In: Proceedings of the 6th Annual Meeting of ISMRM, Sydney, 1998. p 161. 